\title{
Acid-Promoted Reaction of Trifluoromethylated Allyl Alcohols with Arenes. Stereoselective Synthesis of $\mathrm{CF}_{3}$-Alkenes and $\mathrm{CF}_{3}$-Indanes
}

\author{
Anna N. Kazakova, ${ }^{\dagger}$ Roman O. Iakovenko, ${ }^{\dagger}$ Irina A. Boyarskaya, ${ }^{\dagger}$ Valentine G. Nenajdenko, ${ }^{*}{ }^{\dagger}$ \\ and Aleksander V. Vasilyev ${ }^{* \dagger,}, \S$ \\ ${ }^{\dagger}$ Department of Organic Chemistry, Institute of Chemistry, Saint Petersburg State University, Universitetskaya nab., 7/9, Saint \\ Petersburg, 199034, Russia \\ ${ }^{\ddagger}$ Department of Chemistry, Lomonosov Moscow State University, Vorobievy Gory, 1, Moscow, 119899, Russia \\ ${ }^{\S}$ Department of Chemistry, Saint Petersburg State Forest Technical University, Institutsky per., 5, Saint Petersburg, 194021, Russia
}

\section{Supporting Information}

ABSTRACT: Reaction of 4-aryl-1,1,1-trifluorobut-3-en-2-ols $\left[\mathrm{CF}_{3}\right.$-allyl alcohols, $\left.\mathrm{ArCH}=\mathrm{CHCH}(\mathrm{OH}) \mathrm{CF}_{3}\right]$ with arenes under activation with anhydrous $\mathrm{FeCl}_{3}$ or $\mathrm{FSO}_{3} \mathrm{H}$ was studied. We found that the transformation led to trifluoromethylated alkenes $\left[\mathrm{Ar}\left(\mathrm{Ar}^{\prime}\right) \mathrm{CHCH}=\mathrm{CHCF}_{3}\right]$ or 1-trifluoromethylated

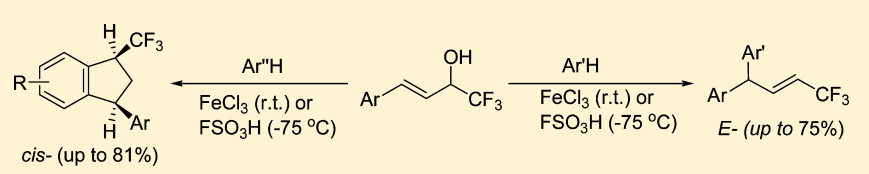

indanes $\left(\mathrm{CF}_{3}\right.$-indanes). The formation of these two types of reaction products strongly depends on the nucleophilicity of the starting arene and the electrophilicity of cationic intermediates generated from $\mathrm{CF}_{3}$-allyl alcohols under reaction conditions. Benzene, anisole, veratrole, and ortho-xylene lead exclusively to $\mathrm{CF}_{3}$-alkenes with an E-configuration. More $\pi$-donating polymethylated arenes (pseudocumene, mesitylene) afford only $\mathrm{CF}_{3}$-indanes with a predominantly cis-orientation of substituents at positions 1 and 3 of the indane ring. Meta- and para-xylenes show an intermediate behavior; they may form both $\mathrm{CF}_{3}$-alkenes and/or $\mathrm{CF}_{3}$-indanes. The mechanisms of the investigated transformations are discussed.

\section{INTRODUCTION}

Fluorinated organic compounds have significant theoretical and practical value in chemistry, biology, medicine, and material science. ${ }^{1,2}$ Incorporation of a fluorinated moiety into a molecule often changes such important parameters as lipophilicity, metabolic activity, and bioavailability. Trifluoromethylated alkenes (styrenes) are compounds of high practical interest. Some derivatives with such fragments have been widely used for organic light-emitting diodes (OLEDs) and other material chemistry applications. ${ }^{3}$ Some trifluoromethylated alkenes attract significant attention due to important biological activity and application in medicine. Incorporation of a $\mathrm{CF}_{3}$ group permits one to design more potent drugs. For example, Tamoxifen (Nolvadex) is the well-known triarylethylene-type antiestrogenic drug, ${ }^{4}$ which is used in the therapy of breast cancer and for the treatment of menstrual disorders. ${ }^{5}$ Panomifene is a trifluoromethylated analogue of Tamoxifen (Figure 1). This drug demonstrated higher anticancer activity; however, quite important is the configuration of the double bond because only one diastereomer can be used.

The electron-withdrawing character of fluorinated groups is another advantage, which allows one to control the selectivity of transformations of fluorinated compounds. $\mathrm{CF}_{3}$-substituted carbocations are very promising, but still very rare, fluorinated species exhibiting high electrophilicity and selectivity. ${ }^{6}$ The present work is a continuation of our investigations on the electrophilic activation of alkenes ${ }^{7}$ and alkynes. ${ }^{8}$ In a preliminary short communication, ${ }^{9}$ we showed that 4-phenyl1,1,1-trifluorobut-3-en-2-ol 1a alkylated selected aromatics to
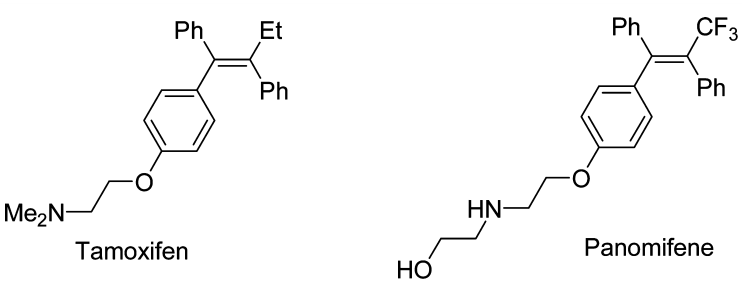

Figure 1. Tamoxifen and panomifene.

afford E-4,4-diaryl-1,1,1-trifluorobut-2-enes 2 under action of various Brønsted or Lewis acids. The best results (highest yields of compounds 2) were obtained using 1 equiv of anhydrous iron trichloride $\mathrm{FeCl}_{3}$ (Scheme 1).

Interaction of $\mathrm{CF}_{3}$-alcohols 1 with Brønsted or Lewis acids proceeds through intermediate formation of various electrophilic species. Thus, coordination of oxygen with a Lewis acid or protonation with a Brønsted acid leads to species $\mathbf{B}$ or $\mathbf{C}$, correspondingly (Scheme 2). Finally, dehydroxylation of

Scheme 1. Reactions of $\mathrm{CF}_{3}$-alcohol 1a with Arenes (Benzene, $o$ - and $m$-Xylenes, Anisole, Veratrole) under the Action of $\mathrm{FeCl}_{3}$ (from ref 9)

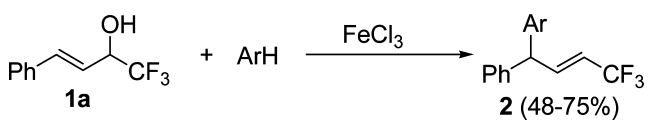

Received: June 25, 2015

Published: September 3, 2015 
Scheme 2

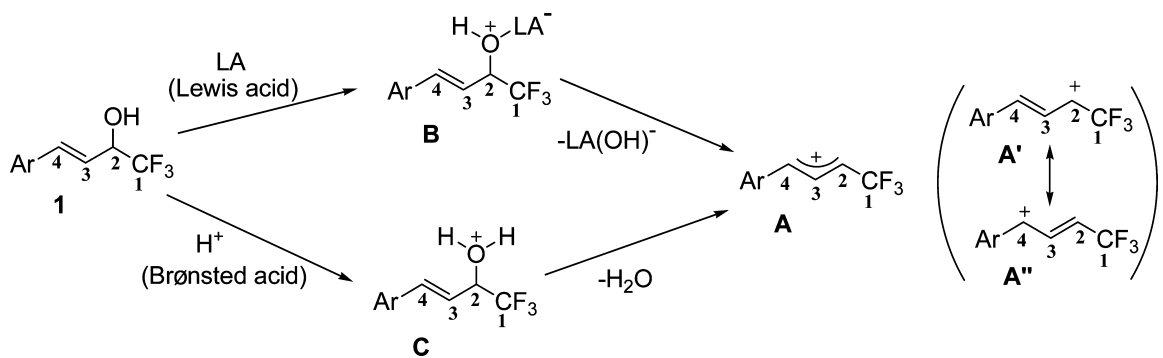

alcohols 1 results in formation of $\mathrm{CF}_{3}$-allyl cations $\mathbf{A}$, having two resonance forms, $\mathbf{A}^{\prime}$ and $\mathbf{A}^{\prime \prime}$, with cationic centers on atoms $\mathrm{C}^{2}$ and $\mathrm{C}^{4}$, respectively. Because of the electron-withdrawing character of the $\mathrm{CF}_{3}$ group. these allyl cations react with arenes through form $\mathrm{A}^{\prime \prime}$ to form a new $\mathrm{C}-\mathrm{C}$ bond at $\mathrm{C}^{4}$ carbon. Intermediates $\mathbf{B}$ or $\mathbf{C}$ also possess highly electrophilic properties and may participate in reactions.

To study this reaction deeper and find its scope and limitations, we decided to investigate a set of $\mathrm{CF}_{3}$-allyl alcohols $\mathbf{1 a}-\mathbf{f}$, bearing various substituents in the aromatic ring (Figure 2) and arenes. Also, we performed a theoretical investigation

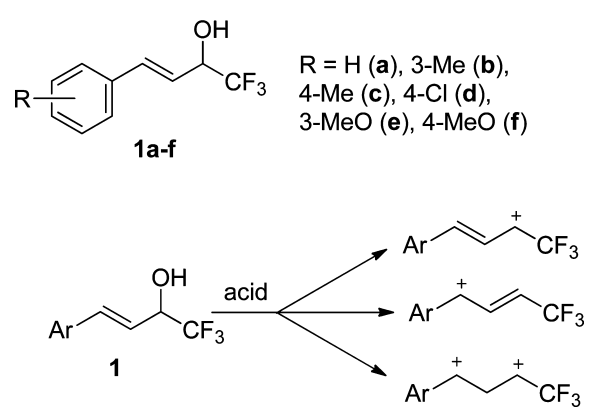

Figure 2. $\mathrm{CF}_{3}$-alcohols $\mathbf{1 a}-\mathbf{f}$ used in this study and their expected acidpromoted reactivity.

(DFT calculations) of intermediate species A, B, C to have their electronic characteristics. It should be noted that one of the most important transformations of allyl cations ${ }^{10}$ is their participation in new carbon-carbon bond forming processes by reactions with arenes, ${ }^{11}$ heteroarenes, ${ }^{12}$ alkenes, ${ }^{13}$ alkynes, ${ }^{14}$ or carbonyl compounds. ${ }^{15}$ To our surprise, reactions of trifluoromethylated allyl cations with C-nucleophiles have not been widely described yet. Structurally related methyl allyl alcohols, bearing a $\mathrm{CH}_{3}$ group instead of a $\mathrm{CF}_{3}$ one, in reactions with (hetero)arenes under the action of Brønsted or Lewis acids afford Friedel-Crafts alkylation products exclusively (predominantly, in some cases) at the $\mathrm{C}^{2}$ carbon (see Scheme 2). ${ }^{11, \mathrm{f}, \mathrm{f}, 12 \mathrm{a}-\mathrm{c}} \mathrm{We}$ expected that introduction of the strong acceptor $\mathrm{CF}_{3}$ group would significantly change the reactivity of such allyl systems compared to previously studied $\mathrm{CH}_{3}$-allyl alcohols.

The main goal of this work was to investigate the reaction of $\mathrm{CF}_{3}$-allyl alcohols $\mathbf{1 a}-\mathbf{f}$ with various arenes under action of Lewis acid $\mathrm{FeCl}_{3}$ or some Brønsted superacids. We expected that electrophilic species formed from alcohols $\mathbf{1}$ in the presence of acids will react with arenes to exhibit at least three types of reaction routes. Three synthons which can describe the chemistry of studied trifluoromethylated allyl alcohols $\mathbf{1}$ are given in Figure 2. They are cationic and dicationic species which are expected to have interesting and rich chemical properties.

\section{RESULTS AND DISCUSSION}

To estimate electronic properties of reaction intermediates, we performed DFT calculations of species A, B, and C, derived from starting alcohols 1a-f (Table 1). Charge distribution, contribution of the atomic orbital into the molecular orbital, and global electrophilicity indices $\omega^{16}$ were calculated. Because of the electron-withdrawing effect of the $\mathrm{CF}_{3}$ group, cations A1-A6 bear a greater positive charge on the carbon $\mathrm{C}^{4}$ compared to $\mathrm{C}^{2}$ (Table 1). Apart from that, atom $\mathrm{C}^{4}$ has a larger LUMO contribution (31-35\%). These data indicate a coincidence of charge and orbital control in reactivity of carbon $\mathrm{C}^{4}$ in cations A. Species $\mathbf{B}$ and $\mathbf{C}$ are characterized by a small positive charge on atom $C^{2}$ and a negative one on $C^{4}$. The LUMO of $\mathbf{B}$ is localized mainly on the iron atom (see the Supporting Information). A higher contribution to the LUMO at carbon $\mathrm{C}^{4}$ compared to $\mathrm{C}^{2}$ is observed for species $\mathrm{C}$. Therefore, charge controlled reactions of species $\mathbf{B}$ and $\mathbf{C}$ should direct nucleophiles at carbon $\mathrm{C}^{2}$. Among these three types of species, the cations A possess the highest electrophilic properties based on the $\omega$ values of $14.1-19.9 \mathrm{eV}$ in comparison with $\omega=6.7-7.5 \mathrm{eV}$ for $\mathbf{B}$ and $\omega=2.9-3.3 \mathrm{eV}$ for $\mathbf{C}$, correspondingly (Table 1 ). The latter may be considered as weaker electrophiles. According to the results of DFT calculations, cation A1 has an absolutely planar geometry. Similarly, species $\mathbf{B 1}$ and $\mathbf{C 1}$ are almost planar (see the Supporting Information). The $\mathrm{C}-\mathrm{O}$ bond in intermediate $\mathbf{C 1}$ is significantly elongated $(1.636 \AA)$ compared to a normal C-O bond in alcohols (1.43 $\AA$ ). In the case of $\mathrm{B1}$, the $\mathrm{C}-\mathrm{O}$ bond is only by $0.03 \AA$ longer $(1.464 \AA$ ) than that in the usual case.

Thus, the obtained calculated data revealed that the atom $\mathrm{C}^{4}$ must be the most reactive center in allyl cations $\mathbf{A}$; on the contrary, species $\mathbf{B}$ and $\mathbf{C}$ should react with nucleophiles with attack to the $C^{2}$ center. In other words, in the first case, the reaction may occur as an $S_{\mathrm{N}} 1^{\prime}$ process, but in the cases of species $\mathbf{B}$ and $\mathbf{C}$, most probably, the reaction should result in the formation of products without an allylic rearrangement. Therefore, reaction conditions (temperature and polarity of solvent) can influence significantly the direction of the reaction to give different types of products.

Having the results of theoretical prediction in hand, we started the investigation of synthetic possibilities of the reaction and their mechanism. $\mathrm{FeCl}_{3}$-promoted reaction of $\mathrm{CF}_{3}$-allyl alcohols 1 with benzene, isomeric xylenes, anisole, and veratrole gave highly regioselectively and stereoselectively $\mathrm{CF}_{3}$-alkenes 2 (Table 2, and Scheme 3). The reaction proceeds as an $S_{N} 1^{\prime}$ type process to form a new $\mathrm{C}-\mathrm{C}$ bond at the $\mathrm{C}^{4}$ position of the starting alcohol. That can be explained by the electronwithdrawing action of the trifluoromethyl group making the 
Table 1. Selected Electronic Characteristics and Calculated Geometries of Species A, B, and C

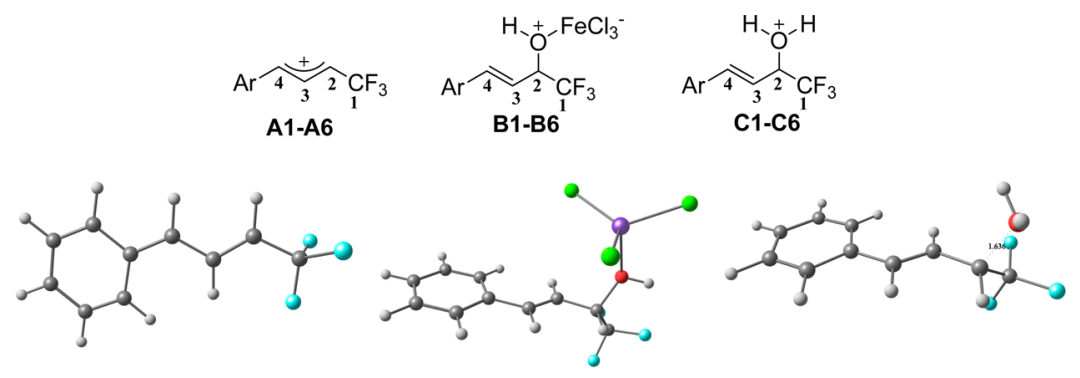

A1

B1

C1

\begin{tabular}{|c|c|c|c|c|c|c|c|c|}
\hline species & $\mathrm{R}$ in $\mathrm{Ar}$ & $E_{\text {Номо }}, \mathrm{eV}$ & $E_{\text {LUMO }}, \mathrm{eV}$ & $\omega,^{a} \mathrm{eV}$ & $q\left(\mathrm{C}^{2}\right){ }^{b} \mathrm{e}$ & $q\left(C^{4}\right), b$ e & $k\left(\mathrm{C}^{2}\right) \mathrm{LUMO},{ }^{c} \%$ & $k\left(\mathrm{C}^{4}\right) \mathrm{LUMO},{ }^{c} \%$ \\
\hline A1 & $\mathrm{H}$ & -12.18 & -8.75 & 16.0 & -0.05 & 0.09 & 24 & 35 \\
\hline A2 & 3-Me & -11.71 & -8.60 & 16.6 & -0.06 & 0.10 & 23 & 35 \\
\hline A3 & 4-Me & -11.89 & -8.43 & 14.9 & -0.07 & 0.08 & 21 & 32 \\
\hline A4 & 4-Cl & -11.88 & -8.66 & 16.4 & -0.06 & 0.08 & 21 & 31 \\
\hline A5 & $3-\mathrm{MeO}$ & -10.96 & -8.56 & 19.9 & -0.10 & 0.10 & 23 & 34 \\
\hline A6 & 4- $\mathrm{MeO}$ & -11.88 & -8.01 & 14.1 & -0.09 & 0.04 & 23 & 35 \\
\hline B1 & $\mathrm{H}$ & -7.12 & -4.57 & 6.7 & 0.02 & -0.11 & $d$ & $d$ \\
\hline B2 & 3-Me & -7.00 & -4.54 & 6.8 & 0.02 & -0.11 & $d$ & $d$ \\
\hline B3 & 4-Me & -6.88 & -4.52 & 6.9 & 0.02 & -0.11 & $d$ & $d$ \\
\hline B4 & $4-\mathrm{Cl}$ & -7.10 & -4.64 & 7.0 & 0.02 & -0.11 & $d$ & d \\
\hline B5 & $3-\mathrm{MeO}$ & -6.69 & -4.52 & 7.3 & 0.02 & -0.11 & $d$ & $d$ \\
\hline B6 & $4-\mathrm{MeO}$ & -6.50 & -4.48 & 7.5 & 0.02 & -0.11 & $d$ & $d$ \\
\hline $\mathrm{C} 1$ & $\mathrm{H}$ & -7.23 & -2.95 & 3.0 & 0.04 & -0.04 & 8 & 25 \\
\hline $\mathrm{C} 2$ & 3-Me & -7.11 & -2.87 & 2.9 & 0.05 & -0.04 & 17 & 12 \\
\hline C3 & 4-Me & -7.03 & -3.11 & 3.3 & 0.04 & -0.02 & 18 & 22 \\
\hline $\mathrm{C} 4$ & $4-\mathrm{Cl}$ & -7.13 & -3.00 & 3.1 & 0.04 & -0.04 & 14 & 20 \\
\hline C5 & $3-\mathrm{MeO}$ & -6.70 & -2.88 & 3.0 & 0.05 & -0.04 & 13 & 13 \\
\hline C6 & 4- $\mathrm{MeO}$ & -6.61 & -2.92 & 3.1 & 0.04 & -0.03 & 9 & 19 \\
\hline
\end{tabular}

${ }^{a}$ Global electrophilicity index $\omega=\left(E_{\mathrm{HOMO}}+E_{\mathrm{LUMO}}\right)^{2} / 8\left(E_{\mathrm{LUMO}}-E_{\mathrm{HOMO}}\right) .{ }^{b}$ Natural charges. ${ }^{c}$ Contribution of atomic orbital into the molecular orbital. ${ }^{d}$ LUMO is mainly localized on iron atom.

Table 2. Reaction of $\mathrm{CF}_{3}$-allyl Alcohols 1 with Arenes, Leading to $\mathrm{CF}_{3}$-alkenes 2

\begin{tabular}{|c|c|c|c|c|c|c|c|}
\hline \multirow[b]{2}{*}{ entry } & \multicolumn{2}{|c|}{ starting materials } & \multirow[b]{2}{*}{ conditions $^{a}$} & \multicolumn{4}{|c|}{ reaction products, $E-2$} \\
\hline & alcohol $1(\mathrm{R}$ in $\mathrm{Ar})$ & arene, $\mathrm{R}^{\prime}$ & & $\mathrm{N}$ & $\mathrm{R}$ & $\mathrm{R}^{\prime}$ & isolated yield, \% \\
\hline 1 & 1a $(\mathrm{R}=\mathrm{H})$ & $\mathrm{H}$ & A & $2 a$ & $\mathrm{H}$ & $\mathrm{H}$ & 65 \\
\hline 2 & 1a $(\mathrm{R}=\mathrm{H})$ & 1,2-diMe & A & $2 b$ & $\mathrm{H}$ & 3,4-diMe & 75 \\
\hline 3 & 1a $(\mathrm{R}=\mathrm{H})$ & 1,3-diMe & A & $2 c$ & $\mathrm{H}$ & 2,4-diMe & 56 \\
\hline 4 & 1a $(\mathrm{R}=\mathrm{H})$ & $\mathrm{MeO}$ & A & $2 d+2 e(4 / 1)$ & $\mathrm{H}$ & $\mathrm{MeO}$ & $72^{b}$ \\
\hline 5 & 1a $(\mathrm{R}=\mathrm{H})$ & 1,2-diMeO & A & $2 f$ & $\mathrm{H}$ & 3,4-diMeO & 48 \\
\hline 6 & $1 b(\mathrm{R}=3-\mathrm{Me})$ & 1,2-diMe & A & $2 \mathrm{~g}$ & 3-Me & 3,4-diMe & 47 \\
\hline 7 & $\mathbf{1 d}(\mathrm{R}=4-\mathrm{Cl})$ & $\mathrm{H}$ & A & $2 \mathrm{~h}$ & 4-Cl & $\mathrm{H}$ & 42 \\
\hline 8 & $\mathbf{1 d}(\mathrm{R}=4-\mathrm{Cl})$ & 1,3-diMe & A & $2 \mathrm{i}$ & $4-\mathrm{Cl}$ & 2,4-diMe & 56 \\
\hline 9 & $\mathbf{1 d}(\mathrm{R}=4-\mathrm{Cl})$ & 1,2-diMeO & A & $2 j$ & $4-\mathrm{Cl}$ & 3,4-diMeO & 43 \\
\hline 10 & 1e $(\mathrm{R}=3-\mathrm{MeO})$ & $\mathrm{H}$ & B & $2 \mathrm{k}$ & 3-MeO & $\mathrm{H}$ & 45 \\
\hline 11 & le $(\mathrm{R}=3-\mathrm{MeO})$ & $\mathrm{MeO}$ & B & $2 \mathbf{l}+2 \mathbf{m}(8 / 1)$ & $3-\mathrm{MeO}$ & $\mathrm{MeO}$ & $63^{b}$ \\
\hline
\end{tabular}

${ }^{a}$ Reactions conditions: $\mathbf{A}-\mathrm{FeCl}_{3}, \mathrm{CH}_{2} \mathrm{Cl}_{2}, 20{ }^{\circ} \mathrm{C}, 1 \mathrm{~h}$, molar ratio 1: $\mathrm{FeCl}_{3}: \mathrm{ArH}=1: 1: 1.1 ; \mathbf{B}-\mathrm{FSO}_{3} \mathrm{H}, \mathrm{CH}_{2} \mathrm{Cl}_{2},-75{ }^{\circ} \mathrm{C}, 2$ h, molar ratio $\mathbf{1}: \mathrm{FSO}_{3} \mathrm{H}: \mathrm{ArH}=1: 80: 3 .{ }^{b}$ Mixture of para- $(\mathbf{2 d}, \mathbf{2 l})$ and ortho- $(\mathbf{2 e}, \mathbf{2 m})$ isomers was formed.

more preferable resonance form $\mathbf{A}^{\prime \prime}$ (Scheme 1). It should be noted that this reaction is $100 \%$ diastereoselective to form $E$ isomers 2 only (configurations were confirmed by NOESY; see the Supporting Information). In ${ }^{1} \mathrm{H}$ NMR, values of constants ${ }^{3} J$ between protons at the carbon-carbon double bond are $15.6-15.7 \mathrm{~Hz}$, which correspond to the E-configuration. This stereochemical result can be explained by high steric demand of both the diarylmethyl group and the trifluoromethyl moiety attached to the formed double bond.

Methoxy substituted alcohols $\mathbf{1 e}, \mathbf{f}$ afforded complex mixtures of oligomeric products in the same reactions under action of $\mathrm{FeCl}_{3}$ (at r.t.) or Brønsted superacid TfOH (at r.t. or $-35^{\circ} \mathrm{C}$ ). It probably happened due to high $\pi$-nucleophilicity of 
Scheme 3

1a
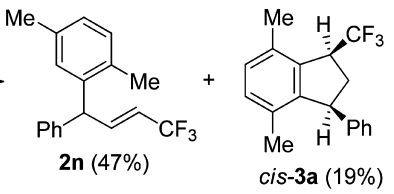

$1 \mathrm{~d}$

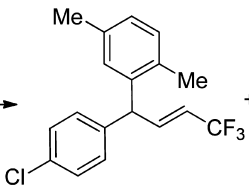

$20(69 \%)$

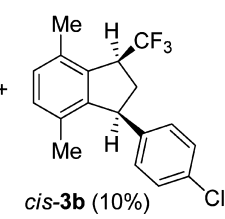

methoxylated aromatic rings trapping intermediate cationic species and leading the reaction into alternative routes at these conditions. We managed to involve $\mathrm{CF}_{3}$-allyl alcohols $\mathbf{1 e , f}$ in the reaction with arenes only at low temperature $-75{ }^{\circ} \mathrm{C}$ in superacid $\mathrm{FSO}_{3} \mathrm{H}$ (entries 10, 11, and see Scheme 6).

To check our hypothesis about the possibility to redirect the reaction, we studied more carefully the reaction with more nucleophilic arenes (xylenes, pseudocumene, and mesitylene) and thiophene. Surprisingly, in the case of $\mathrm{FeCl}_{3}$ activation reaction of alcohols 1a,d with para-xylene, we observed not only formation of alkenes $\mathbf{2 n}, \mathbf{o}$ but also of $\mathrm{CF}_{3}$-indanes $\mathbf{3 a}, \mathbf{b}$, which were isolated as minor products (Scheme 3 ). The corresponding trifluoromethylated indanes were isolated as cisisomers; their configuration was established using NOESY correlations (Figure 3).
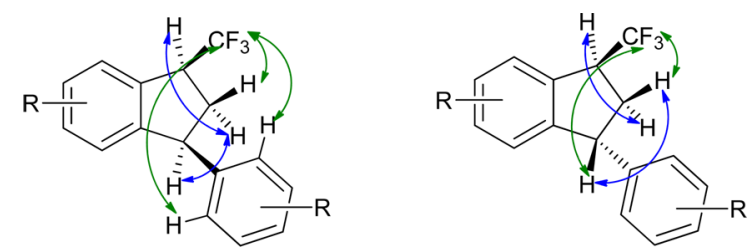

Figure 3. Selected NOESY correlations for cis- and trans-indanes 3 (blue: H-H correlations; green: H-F correlations).

The appearance of compounds 3 reveals the participation of carbon $\mathrm{C}^{2}$ from the initial $\mathrm{CF}_{3}$-alcohols 1 (see Scheme 3 ) in reaction with such $\pi$-donating arenes. The formation of indanes 3 can be explained by a two-step electrophilic substitution at the para-xylene ring. The first step of the reaction is the formation of an allyl substituted arene; however, this is not the alkene 2 because our additional experiments showed that alkenes 2 cannot be cyclized to indanes 3 due to electronwithdrawing destabilization of the formed carbocation by the trifluoromethyl group (Scheme 8). It is quite interesting that, in this reaction, trifluoromethylated alcohols perform as 1,3dicationic synthons.

The indane (indene) fragment is a very important structural unit of a large number of bioactive and pharmaceutically interesting molecules as well as modern catalysts for polymerization. 2-Trifluoromethylated indanes are an important type of indane derivatives, which have shown different biological activities: antiemetic (neurokinin receptor type 1 agonist) ${ }^{17 a}$ and anticancer (pyruvate dehydrogenase kinase (PDHK) inhibitor, ${ }^{17 b}$ growth factor receptor tyrosine kinase inhibitor $\left.{ }^{17 \mathrm{c}}\right)$. Apart from that, recently, we have shown that the trans1,3-diaryl-1-trifluoromethyl indane scaffold is a new core for cannabinoid receptor ligand design. ${ }^{17 \mathrm{~d}}$ However, so far, the existing approaches to trifluoromethylated indanes have some restrictions. ${ }^{18}$ The synthesis proposed in this investigation is quite straightforward to construct highly desirable $\mathrm{CF}_{3}$-indanes 3 from $\mathrm{CF}_{3}$-alcohols 1 and arene in a one-pot sequence.

The reaction of $\mathbf{1} \mathbf{b}$ with para- and meta-xylenes provided exclusively the corresponding trifluoromethylated indanes in moderate yields (Scheme 4 ). In the case of para-xylene, we observed stereoselective formation of indane $3 \mathrm{c}$ having a cisconfiguration, whereas, in the case of meta-xylene, a mixture of cis-/trans-isomers $\mathbf{3 d}$ in the ratio of $1: 3$ was formed. These stereochemical observations can be explained by the more hindered structure of $3 \mathbf{c}$, having an additional methyl group in position 4 of the indane core.

More striking results were obtained with highly nucleophilic pseudocumene and mesitylene (Scheme 5). Alcohols 1a-d gave in the reaction with pseudocumene exclusively cis-indanes $\mathbf{3 e}-\mathbf{h}$. Reactions with mesitylene led to cis-, trans-indanes $\mathbf{3 e}, \mathbf{g}, \mathbf{h}$ (Scheme 5). Formation of these types of indanes showed that a shift of the methyl group in the mesitylene ring takes place (Scheme 5). These results can be explained by protonation of intermediate alkene $\mathbf{D}$; the next step is electrophilic cyclization in cation $\mathbf{E}$, followed by migration of methyl group in species $\mathbf{F}$ and elimination of a proton from the aryl ring, leading finally to indanes 3.

Analogously to $\mathrm{FeCl}_{3}$-promoted synthesis of indanes (Schemes 3-5), alcohol if can be activated with fluorosulfonic acid to form in the reaction with pseudocumene stereoselectively only cis-indane $3 \mathbf{i}$ (Scheme 6). The reaction depends significantly on the reactivity and nature of the aromatic substrate. For example, in the case of reaction with anisole, we observed no formation of indane. Two products $2 p$ and $4 a$ (Scheme 6) were isolated. The first step of the reaction in the case of $4 a$ is participation of atom $C^{2}$. However, $2 p$ is the result of attack of anisole at $\mathrm{C}^{4}$ atom. These observations can be explained by the higher polarization of the anisole molecule having a nonactivated meta-position for electrophilic substitution; therefore, the formation of noncyclized products is more favorable in this case.

\section{Scheme 4}

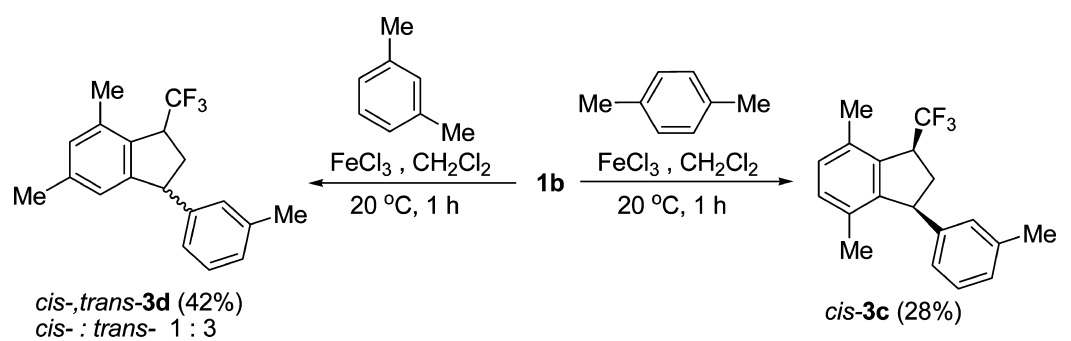




\section{Scheme 5}
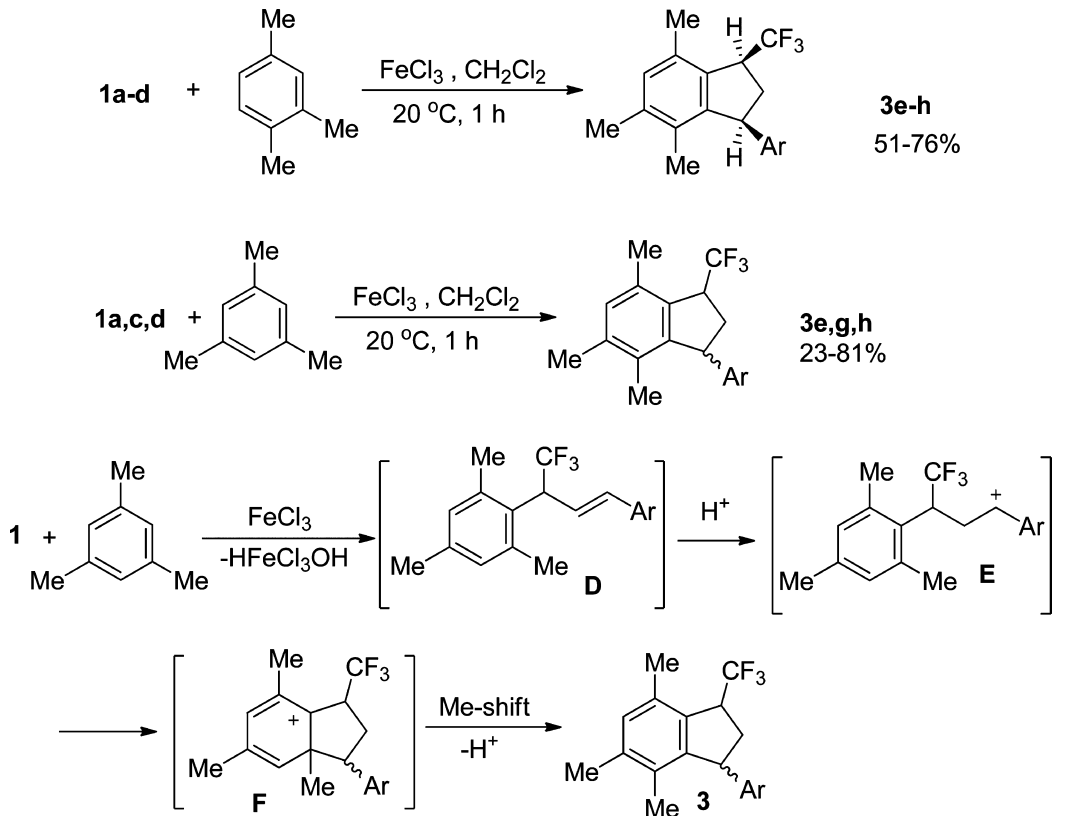

\section{Scheme 6}

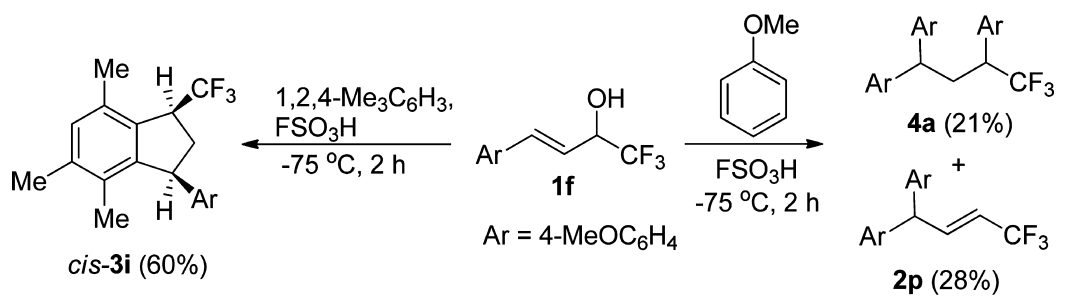

Stereochemical structures of cis- and trans-indanes $\mathbf{3 a - i}$ were determined by NOESY correlations (Figure 3). To have the final confirmation of the configuration, $\mathrm{X}$-ray data for a single crystal of compound cis-3g were obtained (Figure 4). ${ }^{1} \mathrm{H}$ NMR spectra can be also used to determine the configuration. Proton $\mathrm{H}^{3}$ of cis-isomers 3 has a resonance signal at 4.48-4.55 ppm as a doublet with $J 10.2-10.6 \mathrm{~Hz}$; the same proton of transisomers appears as a pseudotriplet at $4.56-4.60 \mathrm{ppm}$ with $J$ 8.6-8.7 Hz. Despite the predominant formation of cis-isomers 3, DFT calculations have shown that the differences between Gibbs energies of cis- and trans-isomers $3 \mathrm{a}$ and $3 \mathrm{~d}$ are 6.4 and $9.6 \mathrm{~kJ} / \mathrm{mol}$, correspondingly, in favor of the trans-isomers (see the Supporting Information). Therefore, the formation of cisindanes is a kinetically controlled process.

We also studied the $\mathrm{FeCl}_{3}$-promoted reaction of $\mathrm{CF}_{3}$ alcohols 1 with thiophene (Scheme 7). It is well-known that thiophene is highly activated toward electrophiles and also rather stable in acidic media compared to other five-membered heterocycles. Another important feature of thiophene chemistry is connected with the significant strain that appeared when an additional five-membered ring is condensed with the thiophene fragment, making such a reaction unfavorable. ${ }^{19}$ Therefore, we expected the absence of indane formation in the case of the reaction with thiophene.

This assumption was found in very good agreement with experiment. When the ratio of alcohols 1 and thiophene was 1 to 1 , mixtures of mono-5 and bis-alkylated products 6 were formed in good total yield (Scheme 7). The reaction proceeds highly stereoselectively and regioselectively in terms of

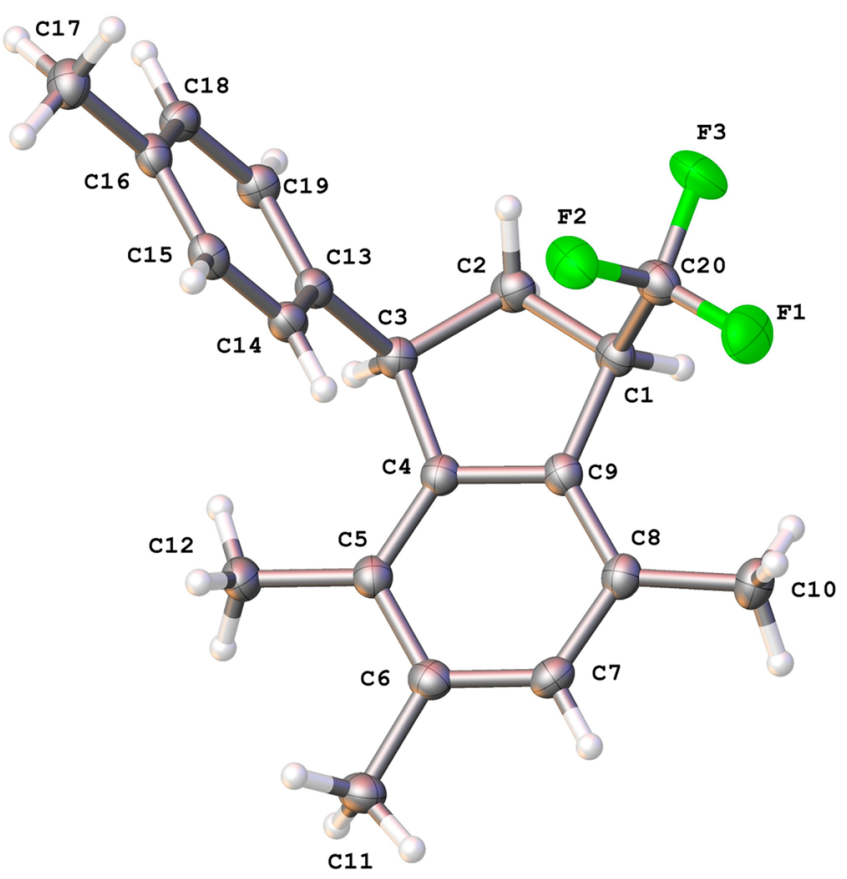

Figure 4. Molecular structure of cis-3g (ellipsoid contour of probability levels is $50 \%$ ).

thiophene substitution (only electrophilic substitution in the $\alpha$-position is observed) and alcohols 1 (reaction at $\mathrm{C}^{4}$ carbon). 
Scheme 7

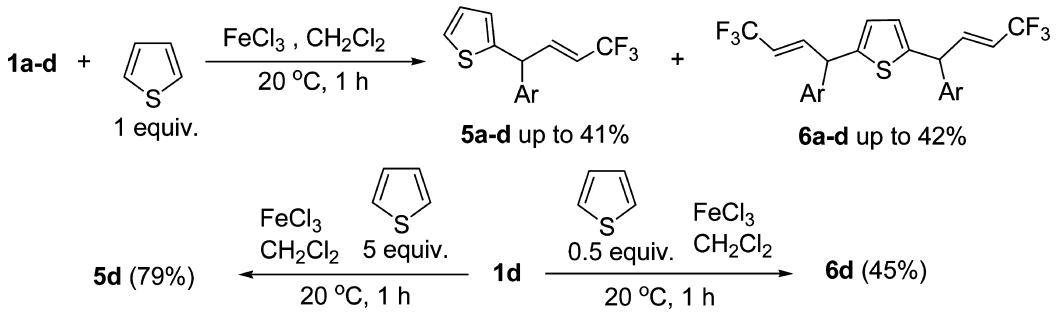

Scheme 8. Possible Mechanism of Acid-Promoted Reaction of 1 with Arenes

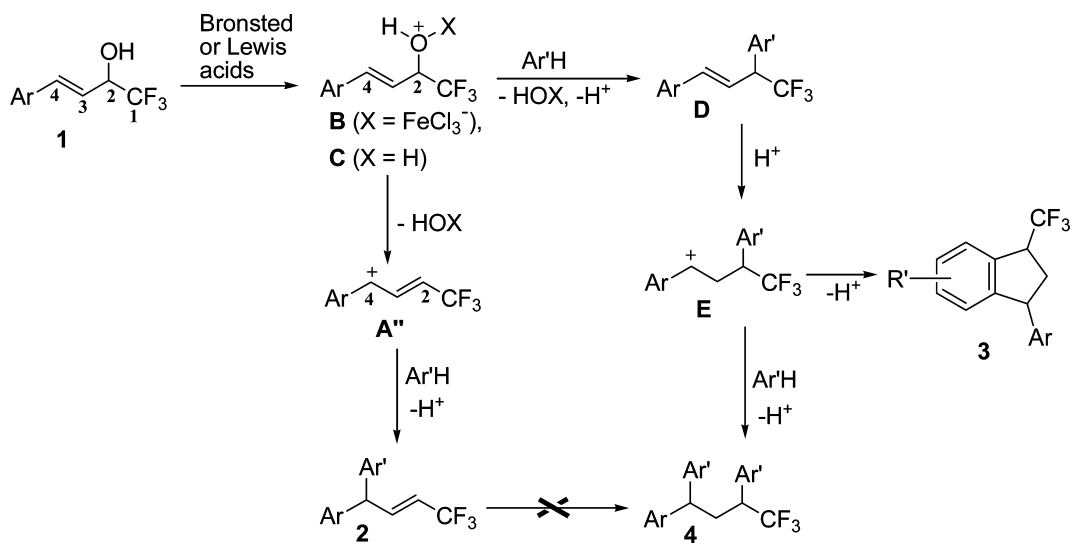

Structures of compounds 5 and $\mathbf{6}$ prove that the $\mathrm{C}^{4}$ atom of resonance form $\mathbf{A}^{\prime \prime}$ (Scheme 2) takes part in alkylation. The reaction can be performed more selectively to provide exclusively either compound $\mathbf{5}$ or $\mathbf{6}$. For example, the reaction of $1 \mathbf{d}$ with an excess of thiophene ( 5 equiv) gave selectively only $5 \mathrm{~d}$, but in the case of the use of 0.5 equiv of thiophene, adduct $6 \mathrm{~d}$ was obtained solely (Scheme 7). The E-configuration of double bonds in alkenes $\mathbf{5}$ or $\mathbf{6}$ was confirmed by NMR (see the Supporting Information). It should be noted that compounds 6 may be formed as mixtures of meso- and $d, l$ isomers, but it is difficult to determine their ratio due to overlapping of signals of isomers in ${ }^{1} \mathrm{H}$ and ${ }^{19} \mathrm{~F}$ NMR spectra (see the Supporting Information).

Summarizing the discussed reactions (Table 2, Schemes 3-7), one may conclude that acid-promoted reaction of alcohols 1 with arenes can proceed by several pathways with participation of both reactive centers at carbons $\mathrm{C}^{4}$ and/or $\mathrm{C}^{2}$ (Scheme 1). Formation of indanes 3 (Schemes 3-6) and products of double arylation $\mathbf{4 a}$ (Scheme 6) reveal the participation at the first step of the reaction of atom $\mathrm{C}^{2}$ of starting alcohol 1. Alkylation of arenes (Table 2, Scheme 3) and thiophene (Scheme 7) may be explained through intermediate generating of an allyl cation reacting highly predominantly at atom $\mathrm{C}^{4}$ (Scheme 2). However, involvement of atom $\mathrm{C}^{2}$ in reactions may prove that species other than $\mathbf{A}$ also take part in these transformations. Most probably these particles have structures $\mathbf{B}$ and $\mathbf{C}$ (Scheme 2), derived from alcohols $\mathbf{1}$ under coordination of $\mathrm{FeCl}_{3}$ on hydroxyl group oxygen or by protonation of this oxygen. Indeed, it should be difficult to eliminate the hydroxyl group from initial alcohol 1 to generate allyl cation $\mathbf{A}$ due to powerful electron-withdrawing properties of group $\mathrm{CF}_{3}$. Intermediates $\mathbf{B}$ and $\mathbf{C}$ may participate in the reaction medium and react with rather nucleophilic electron rich arenes.
On the basis of experimental results and theoretical calculations, we proposed a possible reaction mechanism including multiple pathways of $\mathrm{CF}_{3}$-allyl alcohols 1 with arenes under action of Lewis or Brønsted acids (Scheme 8). Species B and $\mathbf{C}$, having enough electrophilic center on $\mathrm{C}^{2}$, react with rather $\pi$-donating arenes, such as xylenes, pseudocumene, and mesitylene, forming compounds $D$. Further protonation of the formed alkene leads to cation $\mathbf{E}$ (Scheme 8), which may react in two different pathways. The first direction is intramolecular cyclization into indanes 3 . This route is realized when the adjacent ortho-position is quite reactive. Another pathway is the reaction with one more arene molecule, leading to compounds 4 (Scheme 8). It should be noted that compounds 2 in $\mathrm{FeCl}_{3}$ or $\mathrm{FSO}_{3} \mathrm{H}$-promoted reactions are not cyclized into indanes 3 and do not give 4 with arenes, due to deactivation of the double bond to protonation in alkenes 2 by the electron-withdrawing $\mathrm{CF}_{3}$ group. Directions of all of these transformations of alcohols 1 into different products 2-4 depend on the electrophilicity of species A, B, C and nucleophilicity of arenes. In some cases, mixed mechanism can be realized, for example, the reaction of $\mathbf{1}$ with para-xylene, giving mixtures of compounds 2 and 3 (Scheme 3).

\section{CONCLUSIONS}

In conclusion, we have studied the acid-promoted reaction of 4aryl-1,1,1-trifluorobut-3-en-2-ols ( $\mathrm{CF}_{3}$-allyl alcohols) with arenes. It was found that the most efficient activators of this reaction are anhydrous $\mathrm{FeCl}_{3}$ and $\mathrm{FSO}_{3} \mathrm{H}$. The reaction affords efficient stereoselective synthesis of trifluoromethylated alkenes (up to $75 \%$ ) and indanes (up to $81 \%$ ): only $E-C_{3}$-alkenes and predominantly cis- $\mathrm{CF}_{3}$-indanes are formed. Short reaction times, good yields, and simplicity of the reaction procedure are significant advantages of the method. A possible reaction mechanism includes three types of electrophilic species derived from starting alcohols. The formation of two types of reaction 
products depends on the nucleophilicity of arene and the electrophilicity of cationic intermediates, generated from $\mathrm{CF}_{3}$ allyl alcohols under reaction conditions.

\section{EXPERIMENTAL SECTION}

The NMR spectra of solutions of compounds in $\mathrm{CDCl}_{3}$ were recorded at 400, 376, and $100 \mathrm{MHz}$ for ${ }^{1} \mathrm{H},{ }^{19} \mathrm{~F}$, and ${ }^{13} \mathrm{C}$ NMR, respectively, at $25{ }^{\circ} \mathrm{C}$. The residual proton-solvent peak $\mathrm{CDCl}_{3}(\delta 7.26 \mathrm{ppm})$ for ${ }^{1} \mathrm{H}$ NMR spectra and the carbon signal of $\mathrm{CDCl}_{3}(\delta 77.0 \mathrm{ppm})$ for ${ }^{13} \mathrm{C}$ NMR spectra were used as references. Chromato-mass-spectrometry data were obtained at a capillary column $(30 \mathrm{~m} \times 0.32 \mathrm{~mm})$, with the thickness of the stationary phase being $1.25 \mu \mathrm{m}$. The preparative reactions were monitored by thin-layer chromatography carried out on silica gel plates, using UV light for detection. Preparative TLC was performed on silica gel $(5-40 \mu \mathrm{m})$ with a petroleum ether-ethyl acetate mixture eluation.

A suitable crystal was selected and studied on the diffractometer for $\mathrm{X}$-ray analysis. The crystal was kept at 100(2) K during data collection. Using Olex2, ${ }^{20}$ the structure was solved with the ShelXS ${ }^{20}$ structure solution program using direct methods and refined with the ShelXL refinement package using least-squares minimization. CCDC 1406002 (cis-3g) contain the supplementary crystallographic data, which can be obtained free of charge at www.ccdc.cam.ac.uk/conts/retrieving.htmL or from the Cambridge Crystallographic Data Centre, 12 Union Road, Cambridge CB2 1EZ, U.K.; Fax: (internat.) + 44-1223-336-033; Email: deposit@ccdc.cam.ac.uk.

All computations has been carried out at the DFT/HF hybrid level of theory using Becke's three-parameter hybrid exchange functional in combination with the gradient-corrected correlation functional of Lee, Yang, and Parr (B3LYP) by using Gaussian 2003 program packages. ${ }^{21}$ The geometries optimization were performed using the 6-311+G$(2 \mathrm{~d}, 2 \mathrm{p})$ basis set The Hessian matrix was calculated analytically for the optimized structures in order to prove the location of correct minima (no imaginary frequencies) and to estimate the thermodynamic parameters. Enthalpies and Gibbs free energies were calculated for 25 ${ }^{\circ} \mathrm{C}$.

Starting 4-Aryl-1,1,1-trufluorobut-3-en-2-ols (1a-f). The title compounds were obtained by carbonyl reduction of the corresponding 4-aryl-1,1,1-trifluorobut-3-en-2-ones according to the known procedure. ${ }^{22}$ The properties of compounds $\mathbf{1 a}, \mathbf{c}, \mathbf{d}$ were given in our preliminary communication. ${ }^{9}$ Their NMR data matched those previously reported. ${ }^{23}$

(E)-1,1,1-Trifluoro-4-(3-methylphenyl)but-3-en-2-ol (1b). Yield $288 \mathrm{mg}$, 96\%. Yellow oil. ${ }^{1} \mathrm{H} \mathrm{NMR}\left(\mathrm{CDCl}_{3}, 400 \mathrm{MHz}\right) \delta$, ppm: 2.34 (br. s, $1 \mathrm{H}, \mathrm{OH}$ ), $2.36\left(\mathrm{~s}, 3 \mathrm{H}, \mathrm{CH}_{3}\right), 4.63$ (pseudo-quintet d, $1 \mathrm{H}, J$ $\left.=6.6 \mathrm{~Hz}, J_{\mathrm{H}-\mathrm{F}}=6.6 \mathrm{~Hz}, J=1.0 \mathrm{~Hz}\right), 6.19(\mathrm{dd}, 1 \mathrm{H}, J=16.0 \mathrm{~Hz}, J=6.6$ $\mathrm{Hz}), 6.83(\mathrm{~d}, 1 \mathrm{H}, J=16.0 \mathrm{~Hz}), 7.12-7.14(\mathrm{~m}, 1 \mathrm{H}), 7.22-7.27(\mathrm{~m}$, $3 \mathrm{H}) .{ }^{13} \mathrm{C}$ NMR $\left(\mathrm{CDCl}_{3}, 100 \mathrm{MHz}\right) \delta$, ppm: $21.4\left(\mathrm{CH}_{3}\right), 71.8\left(\mathrm{q}, \mathrm{C}^{2}\right.$, $\left.J_{\mathrm{C}-\mathrm{F}}=32.3 \mathrm{~Hz}\right), 120.6\left(\mathrm{q}, \mathrm{C}^{3}, J_{\mathrm{C}-\mathrm{F}}=1.7 \mathrm{~Hz}\right), 124.4\left(\mathrm{q}, \mathrm{C}^{1}, J_{\mathrm{C}-\mathrm{F}}=281.9\right.$ $\mathrm{Hz}), 124.2,127.7,128.8,129.7,135.4,136.6\left(\mathrm{C}^{4}\right), 138.5 .{ }^{19} \mathrm{~F}$ NMR $\left(\mathrm{CDCl}_{3}, 376 \mathrm{MHz}\right) \delta$, ppm: $-79.07\left(\mathrm{~d}, \mathrm{CF}_{3}, J_{\mathrm{H}-\mathrm{F}}=6.6 \mathrm{~Hz}\right)$. HRMS (ESI): $\mathrm{C}_{11} \mathrm{H}_{12} \mathrm{~F}_{3} \mathrm{O}$ found $217.0837[\mathrm{M}+\mathrm{H}]^{+}$; calcd. 217.0840.

(E)-1,1,1-Trifluoro-4-(3-methoxyphenyl)but-3-en-2-ol (1e). Yield $280 \mathrm{mg}$, 93\%. Yellow oil. ${ }^{1} \mathrm{H} \mathrm{NMR}\left(\mathrm{CDCl}_{3}, 400 \mathrm{MHz}\right) \delta$, ppm: 2.48 (br. s, $1 \mathrm{H}, \mathrm{OH}), 3.84\left(\mathrm{~s}, 3 \mathrm{H}, \mathrm{CH}_{3}\right), 4.64$ (pseudo-quintet d, $1 \mathrm{H}, J=$ $\left.6.5 \mathrm{~Hz}, J_{\mathrm{H}-\mathrm{F}}=6.5 \mathrm{~Hz}, J=0.8 \mathrm{~Hz}\right), 6.21(\mathrm{dd}, 1 \mathrm{H}, J=15.9 \mathrm{~Hz}, J=6.5$ $\mathrm{Hz}), 6.84(\mathrm{~d}, 1 \mathrm{H}, J=15.9 \mathrm{~Hz}), 6.87-7.30(\mathrm{~m}, 4 \mathrm{H}) .{ }^{13} \mathrm{C} \mathrm{NMR}$ $\left(\mathrm{CDCl}_{3}, 100 \mathrm{MHz}\right) \delta$, ppm: $55.4\left(\mathrm{CH}_{3}\right), 71.7\left(\mathrm{q}, \mathrm{C}^{2}, J_{\mathrm{C}-\mathrm{F}}=32.3 \mathrm{~Hz}\right)$, $112.8,114.6,119.7,121.1\left(\mathrm{C}^{3}\right), 124.4\left(\mathrm{q}, \mathrm{C}^{1}, J_{\mathrm{C}-\mathrm{F}}=281.8 \mathrm{~Hz}\right), 129.9$, $136.3\left(\mathrm{C}^{4}\right), 136.9,160.0 .{ }^{19} \mathrm{~F} \mathrm{NMR}\left(\mathrm{CDCl}_{3}, 376 \mathrm{MHz}\right) \delta$, ppm: $-79.04\left(\mathrm{~d}, \mathrm{CF}_{3}, J_{\mathrm{H}-\mathrm{F}}=6.5 \mathrm{~Hz}\right)$. HRMS (ESI): $\mathrm{C}_{11} \mathrm{H}_{12} \mathrm{~F}_{3} \mathrm{O}_{2}$ found 233.0811 $[\mathrm{M}+\mathrm{H}]^{+}$; calcd. 233.0789.

(E)-1,1,1-Trifluoro-4-(4-methoxyphenyl)but-3-en-2-ol (1f). ${ }^{24}$ Yield $300 \mathrm{mg}, 99 \%$. Colorless solid, mp $38-40{ }^{\circ} \mathrm{C} .{ }^{1} \mathrm{H}$ NMR $\left(\mathrm{CDCl}_{3}, 400\right.$ $\mathrm{MHz}) \delta$, ppm: 2.45 (br. s, $1 \mathrm{H}, \mathrm{OH}), 3.82\left(\mathrm{~s}, 3 \mathrm{H}, \mathrm{CH}_{3}\right), 4.64$ (pseudoquintet, $\left.1 \mathrm{H}, J=6.5 \mathrm{~Hz}, J_{\mathrm{H}-\mathrm{F}}=6.5 \mathrm{~Hz}\right), 6.06(\mathrm{dd}, 1 \mathrm{H}, J=15.9 \mathrm{~Hz}, J=$ $6.5 \mathrm{~Hz}), 6.78(\mathrm{~d}, 1 \mathrm{H}, J=15.9 \mathrm{~Hz}), 6.86-6.90(\mathrm{~m}, 2 \mathrm{H}), 7.34-7.37(\mathrm{~m}$, $2 \mathrm{H}) .{ }^{19} \mathrm{~F} \mathrm{NMR}\left(\mathrm{CDCl}_{3}, 376 \mathrm{MHz}\right) \delta$, ppm: $-79.10\left(\mathrm{~d}, \mathrm{CF}_{3}, J_{\mathrm{H}-\mathrm{F}}=6.5\right.$ $\mathrm{Hz})$.
General Procedure for the Reaction of 1 with Arenes and Thiophene under Action of $\mathrm{FeCl}_{3}$. Synthesis of 2, 3, 5, and 6 . Anhydrous $\mathrm{FeCl}_{3}(0.3 \mathrm{mmol})$ was added to a solution of alcohol $\mathbf{1}(0.3$ $\mathrm{mmol})$ and arene $(0.32 \mathrm{mmol})$ or thiophene $(0.34 \mathrm{mmol})$ in anhydrous dichloromethane $(1 \mathrm{~mL})$. The mixture was stirred at room temperature for $1 \mathrm{~h}$ and then quenched with a saturated aqueous solution of $\mathrm{NH}_{4} \mathrm{Cl}$. The aqueous layer was extracted with $\mathrm{CHCl}_{3}(2 \times$ $50 \mathrm{~mL}$ ), and the combined organic layers were dried over $\mathrm{Na}_{2} \mathrm{SO}_{4}$ and concentrated under reduced pressure. The crude mixture was purified by preparative TLC on silica gel, using petroleum ether or petroleum ether-EtOAc mixtures (95:5 to 9:1) as an eluent.

General Procedure for the Reaction of 1 with Arenes in $\mathrm{FSO}_{3} \mathrm{H}$. Synthesis of 2, 3, 4. Alcohol $1(0.26 \mathrm{mmol})$ was added to mixture of $\mathrm{FSO}_{3} \mathrm{H}$ (at $\left.-75{ }^{\circ} \mathrm{C}\right)(1 \mathrm{~mL}), \mathrm{CH}_{2} \mathrm{Cl}_{2}(1-3 \mathrm{~mL})$ with benzene $(0.69 \mathrm{~mL})$, or another arene $(0.28 \mathrm{mmol})$. The reaction mixture was magnetically stirred for $2 \mathrm{~h}$. The reaction mixture was poured into frozen concentrated aqueous $\mathrm{HCl}(10 \mathrm{~mL})$, diluted with water $(20 \mathrm{~mL})$, and then extracted with chloroform $(2 \times 40 \mathrm{~mL})$. The extracts were combined, washed with water, a saturated aqueous solution of $\mathrm{NaHCO}_{3}$, and water again, and dried over $\mathrm{Na}_{2} \mathrm{SO}_{4}$. The solvent was distilled off under reduced pressure. The crude mixture was purified by preparative TLC on silica gel, using petroleum etherEtOAc mixtures $(9: 1)$ as an eluent.

(E)-1,1,1-Trifluoro-4,4-diphenylbut-2-ene (2a). ${ }^{9}$ In this case, the reaction was performed with the compound 1a $(0.3 \mathrm{mmol})$ and benzene $(14.8 \mathrm{mmol})$. Yield $50 \mathrm{mg}, 65 \%$. Colorless oil. ${ }^{1} \mathrm{H}$ NMR $\left(\mathrm{CDCl}_{3}, 400 \mathrm{MHz}\right) \delta$, ppm: $4.84\left(\mathrm{dqd}, 1 \mathrm{H}, J=6.8 \mathrm{~Hz}, J=1.6 \mathrm{~Hz}, J_{\mathrm{H}-\mathrm{F}}\right.$ $=2.0 \mathrm{~Hz}), 5.50\left(\mathrm{dqd}, 1 \mathrm{H}, \mathrm{C}^{2} \mathrm{H}, J=15.6 \mathrm{~Hz}, J_{\mathrm{H}-\mathrm{F}}=6.4 \mathrm{~Hz}, J=1.6 \mathrm{~Hz}\right)$, $6.87\left(\mathrm{ddq}, 1 \mathrm{H}, J=15.6 \mathrm{~Hz}, J=6.8 \mathrm{~Hz}, J_{\mathrm{H}-\mathrm{F}}=2.0 \mathrm{~Hz}\right), 7.16-7.36(\mathrm{~m}$, $10 \mathrm{H}){ }^{19} \mathrm{~F} \mathrm{NMR}\left(\mathrm{CDCl}_{3}, 376 \mathrm{MHz}\right) \delta, \mathrm{ppm}:-63.70\left(\mathrm{dt}, \mathrm{CF}_{3}, J_{\mathrm{H}-\mathrm{F}}=\right.$ $\left.6.4 \mathrm{~Hz}, J_{\mathrm{H}-\mathrm{F}}=2.0 \mathrm{~Hz}\right)$.

(E)-1,1,1-Trifluoro-4-(3,4-dimethylphenyl)-4-phenylbut-2-ene (2b). ${ }^{9}$ Yield $64 \mathrm{mg}, 75 \%$. Colorless oil. ${ }^{1} \mathrm{H}$ NMR $\left(\mathrm{CDCl}_{3}, 400 \mathrm{MHz}\right) \delta$, ppm: $2.26\left(\mathrm{~s}, 6 \mathrm{H}, 2 \mathrm{CH}_{3}\right), 4.78(\mathrm{~m}, 1 \mathrm{H}), 5.52(\mathrm{dqd}, 1 \mathrm{H}, J=15.6 \mathrm{~Hz}$, $\left.J_{\mathrm{H}-\mathrm{F}}=6.4 \mathrm{~Hz}, J=1.7 \mathrm{~Hz}\right), 6.88\left(\mathrm{ddq}, 1 \mathrm{H}, J=15.6 \mathrm{~Hz}, J=6.8 \mathrm{~Hz}, J_{\mathrm{H}-\mathrm{F}}\right.$ $=2.0 \mathrm{~Hz}), 6.90-6.92(\mathrm{~m}, 1 \mathrm{H}), 6.95(\mathrm{~s}, 1 \mathrm{H}), 7.11(\mathrm{~d}, 1 \mathrm{H}, J=7.6 \mathrm{~Hz})$, $7.17-7.19(\mathrm{~m}, 2 \mathrm{H}), 7.25-7.36(\mathrm{~m}, 3 \mathrm{H}) .{ }^{19} \mathrm{~F} \mathrm{NMR}\left(\mathrm{CDCl}_{3}, 376\right.$ $\mathrm{MHz}) \delta$, ppm: $-63.60\left(\mathrm{dt}, \mathrm{CF}_{3}, J_{\mathrm{H}-\mathrm{F}}=6.4 \mathrm{~Hz}, J_{\mathrm{H}-\mathrm{F}}=2.0 \mathrm{~Hz}\right)$.

(E)-1,1,1-Trifluoro-4-(2,4-dimethylphenyl)-4-phenylbut-2-ene (2c). ${ }^{9}$ Yield $48 \mathrm{mg}, 56 \%$. Colorless oil. ${ }^{1} \mathrm{H} \mathrm{NMR}\left(\mathrm{CDCl}_{3}, 400 \mathrm{MHz}\right) \delta$, ppm: 2.22 (s, 3H, $\left.\mathrm{CH}_{3}\right), 2.33\left(\mathrm{~s}, 3 \mathrm{H}, \mathrm{CH}_{3}\right), 4.98(\mathrm{~m}, 1 \mathrm{H}), 5.36(\mathrm{dqd}$, $\left.1 \mathrm{H}, J=15.6 \mathrm{~Hz}, J_{\mathrm{H}-\mathrm{F}}=6.4 \mathrm{~Hz}, J=1.8 \mathrm{~Hz}\right), 6.87(\mathrm{ddq}, 1 \mathrm{H}, J=15.6 \mathrm{~Hz}$, $\left.J=6.0 \mathrm{~Hz}, J_{\mathrm{H}-\mathrm{F}}=2.0 \mathrm{~Hz}\right), 6.95-7.03(\mathrm{~m}, 3 \mathrm{H}), 7.11-7.13(\mathrm{~m}, 2 \mathrm{H})$, $7.24-7.34(\mathrm{~m}, 3 \mathrm{H}) .{ }^{19} \mathrm{~F}$ NMR $\left(\mathrm{CDCl}_{3}, 376 \mathrm{MHz}\right) \delta$, ppm: -63.53 $\left(\mathrm{dt}, \mathrm{CF}_{3}, J_{\mathrm{H}-\mathrm{F}}=6.4 \mathrm{~Hz}, J_{\mathrm{H}-\mathrm{F}}=2.0 \mathrm{~Hz}\right)$.

(E)-1,1,1-Trifluoro-4-(4-methoxyphenyl)-4-phenylbut-2-ene (2d). ${ }^{9}$ Yield $50 \mathrm{mg}$, 58\%. Colorless oil. ${ }^{1} \mathrm{H}$ NMR $\left(\mathrm{CDCl}_{3}, 400 \mathrm{MHz}\right) \delta$, ppm: $3.80\left(\mathrm{~s}, 3 \mathrm{H}, \mathrm{CH}_{3}\right), 4.79-4.81(\mathrm{~m}, 1 \mathrm{H}), 5.49(\mathrm{dqd}, 1 \mathrm{H}, J=15.6 \mathrm{~Hz}$, $\left.J_{\mathrm{H}-\mathrm{F}}=6.4 \mathrm{~Hz}, J=1.6 \mathrm{~Hz}\right), 6.85\left(\mathrm{ddq}, 1 \mathrm{H}, J=15.6 \mathrm{~Hz}, J=6.4 \mathrm{~Hz}, J_{\mathrm{H}-\mathrm{F}}\right.$ $=2.0 \mathrm{~Hz}), 6.89(\mathrm{~d}, 2 \mathrm{H}, J=8.5 \mathrm{~Hz}), 7.08(\mathrm{~d}, 2 \mathrm{H}, J=8.5 \mathrm{~Hz}), 7.16(\mathrm{~d}$, $2 \mathrm{H}, J=7.2 \mathrm{~Hz}), 7.24-7.36(\mathrm{~m}, 3 \mathrm{H}) .{ }^{19} \mathrm{~F} \mathrm{NMR}\left(\mathrm{CDCl}_{3}, 376 \mathrm{MHz}\right) \delta$, ppm: $-63.63 \mathrm{dt}\left(\mathrm{CF}_{3}, J_{\mathrm{H}-\mathrm{F}}=6.4 \mathrm{~Hz}, J_{\mathrm{H}-\mathrm{F}}=2.0 \mathrm{~Hz}\right)$.

(E)-1,1,1-Trifluoro-4-(2-methoxyphenyl)-4-phenylbut-2-ene (2e). ${ }^{9}$ Yield $12 \mathrm{mg}, 14 \%$. Pale yellow oil. ${ }^{1} \mathrm{H}$ NMR $\left(\mathrm{CDCl}_{3}, 400 \mathrm{MHz}\right) \delta$, ppm: $3.78\left(\mathrm{~s}, 3 \mathrm{H}, \mathrm{CH}_{3}\right), 5.25-5.26(\mathrm{~m}, 1 \mathrm{H}), 5.44(\mathrm{dqd}, 1 \mathrm{H}, J=15.6$ $\left.\mathrm{Hz}, J_{\mathrm{H}-\mathrm{F}}=6.4 \mathrm{~Hz}, J=1.5 \mathrm{~Hz}\right), 6.85(\mathrm{ddq}, 1 \mathrm{H}, J=15.6 \mathrm{~Hz}, J=6.4 \mathrm{~Hz}$, $\left.J_{\mathrm{H}-\mathrm{F}}=2.0 \mathrm{~Hz}\right), 6.88-6.95(\mathrm{~m}, 2 \mathrm{H}), 7.04(\mathrm{dd}, 1 \mathrm{H}, J=7.5 \mathrm{~Hz}, J=1.3$ $\mathrm{Hz}), 7.16-7.18(\mathrm{~m}, 2 \mathrm{H}), 7.22-7.33(\mathrm{~m}, 4 \mathrm{H}) .{ }^{19} \mathrm{~F} \mathrm{NMR}\left(\mathrm{CDCl}_{3}, 376\right.$ $\mathrm{MHz}) \delta$, ppm: $-63.55\left(\mathrm{dt}, \mathrm{CF}_{3}, J_{\mathrm{H}-\mathrm{F}}=6.4 \mathrm{~Hz}, J_{\mathrm{H}-\mathrm{F}}=2.0 \mathrm{~Hz}\right)$.

(E)-1,1,1-Trifluoro-4-(3,4-dimethoxyphenyl)-4-phenylbut-2-ene (2f). ${ }^{9}$ Yield $46 \mathrm{mg}$, $48 \%$. Colorless solid, $\mathrm{mp} 67-68{ }^{\circ} \mathrm{C}(\mathrm{MeOH}) .{ }^{1} \mathrm{H}$ NMR $\left(\mathrm{CDCl}_{3}, 400 \mathrm{MHz}\right) \delta$, ppm: $3.82\left(\mathrm{~s}, 3 \mathrm{H}, \mathrm{CH}_{3}\right), 3.87(\mathrm{~s}, 3 \mathrm{H}$, $\left.\mathrm{CH}_{3}\right), 4.79(\mathrm{~m}, 1 \mathrm{H}), 5.50\left(\mathrm{dqd}, 1 \mathrm{H}, J=15.6 \mathrm{~Hz}, J_{\mathrm{H}-\mathrm{F}}=6.3 \mathrm{~Hz}, J=1.2\right.$ $\mathrm{Hz}), 6.65(\mathrm{~d}, 1 \mathrm{H}, J=1.6 \mathrm{~Hz}), 6.70(\mathrm{dd}, 1 \mathrm{H}, J=8.2 \mathrm{~Hz}, J=1.6 \mathrm{~Hz})$, $6.84(\mathrm{~d}, 1 \mathrm{H}, J=8.2 \mathrm{~Hz}), 6.84\left(\mathrm{ddq}, 1 \mathrm{H}, J=15.6 \mathrm{~Hz}, J=6.8 \mathrm{~Hz}, J_{\mathrm{H} \cdot \mathrm{F}}=\right.$ $2.0 \mathrm{~Hz}$ ), $7.16(\mathrm{~d}, 2 \mathrm{H}, J=7.2 \mathrm{~Hz}), 7.25-7.36(\mathrm{~m}, 3 \mathrm{H}) .{ }^{19} \mathrm{~F}$ NMR $\left(\mathrm{CDCl}_{3}, 376 \mathrm{MHz}\right) \delta$, ppm: $-63.63 \mathrm{dt}\left(\mathrm{CF}_{3}, J_{\mathrm{H}-\mathrm{F}}=6.3 \mathrm{~Hz}, J_{\mathrm{H}-\mathrm{F}}=2.0\right.$ $\mathrm{Hz})$.

(E)-1,1,1-Trifluoro-4-(3,4-dimethylphenyl)-4-(3-methylphenyl)but-2-ene (2g). Yield $46 \mathrm{mg}, 48 \%$. Pale yellow oil. ${ }^{1} \mathrm{H} \mathrm{NMR}\left(\mathrm{CDCl}_{3}\right.$ 
$400 \mathrm{MHz}) \delta$, ppm: 2.25 (s, $\left.6 \mathrm{H}, 2 \mathrm{CH}_{3}\right), 2.33\left(\mathrm{~s}, 3 \mathrm{H}, \mathrm{CH}_{3}\right), 4.72-4.73$ $(\mathrm{m}, 1 \mathrm{H}), 5.51\left(\mathrm{dqd}, 1 \mathrm{H}, J=15.6 \mathrm{~Hz}, J_{\mathrm{H}-\mathrm{F}}=6.4 \mathrm{~Hz}, J=1.2 \mathrm{~Hz}\right), 6.85$ $\left(\mathrm{ddq}, 1 \mathrm{H}, J=15.6 \mathrm{~Hz}, J=6.7 \mathrm{~Hz}, J_{\mathrm{H}-\mathrm{F}}=2.0 \mathrm{~Hz}\right), 6.89-6.90(\mathrm{~m}, 1 \mathrm{H})$, $6.93(\mathrm{~s}, 1 \mathrm{H}), 6.96-6.98(\mathrm{~m}, 1 \mathrm{H}), 6.98(\mathrm{~s}, 1 \mathrm{H}), 7.07(\mathrm{~d}, 1 \mathrm{H}, J=7.7$ $\mathrm{Hz}), 7.10(\mathrm{~d}, 1 \mathrm{H}, J=7.7 \mathrm{~Hz}), 7.22(\mathrm{t}, 1 \mathrm{H}, J=7.7 \mathrm{~Hz}) .{ }^{13} \mathrm{C}$ NMR $\left(\mathrm{CDCl}_{3}, 100 \mathrm{MHz}\right) \delta$, ppm: $19.5\left(\mathrm{CH}_{3}\right), 20.0\left(\mathrm{CH}_{3}\right), 21.6\left(\mathrm{CH}_{3}\right)$, $52.5\left(\mathrm{C}^{4}\right), 120.1\left(\mathrm{q}, \mathrm{C}^{2}, J_{\mathrm{C}-\mathrm{F}}=33.4 \mathrm{~Hz}\right), 123.4\left(\mathrm{q}, \mathrm{C}^{1}, J_{\mathrm{C}-\mathrm{F}}=269.5 \mathrm{~Hz}\right)$, $125.6,126.0,127.9,128.7,129.3,129.9,130.1,135.5,137.1,138.5$, 138.8, 141.5, $142.8\left(\mathrm{q}, \mathrm{C}^{3}, J_{\mathrm{C}-\mathrm{F}}=6.3 \mathrm{~Hz}\right) .{ }^{19} \mathrm{~F} \mathrm{NMR}\left(\mathrm{CDCl}_{3}, 376\right.$ $\mathrm{MHz}) \delta$, ppm: $-63.58\left(\mathrm{dt}, \mathrm{CF}_{3}, J_{\mathrm{H}-\mathrm{F}}=6.4 \mathrm{~Hz}, J_{\mathrm{H}-\mathrm{F}}=2.0 \mathrm{~Hz}\right) . \mathrm{MS}$ (GC-MS, EI), $m / z$, ( ( $\left.\mathrm{I}_{\text {rel, }} \%\right): 304(80)[\mathrm{M}]^{+}, 289(100), 269(5), 235$ (8), 212 (13), 197 (33), 179 (10), 165 (5), 144 (13), 129 (28), 110 (18), 91 (10), 77 (10), 51 (5). HRMS (ESI): $\mathrm{C}_{19} \mathrm{H}_{20} \mathrm{~F}_{3}$ found $305.1514[\mathrm{M}+\mathrm{H}]^{+}$; calcd. 305.1517 .

(E)-4-(4-Chlorophenyl)-1,1,1-trifluoro-4-phenylbut-2-ene (2h). Yield $30 \mathrm{mg}, 42 \%$. Pale yellow oil. ${ }^{1} \mathrm{H}$ NMR $\left(\mathrm{CDCl}_{3}, 400 \mathrm{MHz}\right) \delta$, ppm: $4.81-4.83(\mathrm{~m}, 1 \mathrm{H}), 6.50\left(\mathrm{dqd}, 1 \mathrm{H}, J=15.6 \mathrm{~Hz}, J_{\mathrm{H}-\mathrm{F}}=6.0 \mathrm{~Hz}, J\right.$ $=1.5 \mathrm{~Hz}), 6.83\left(\mathrm{ddq}, 1 \mathrm{H}, J=15.6 \mathrm{~Hz}, J=6.8 \mathrm{~Hz}, J_{\mathrm{H}-\mathrm{F}}=2.0 \mathrm{~Hz}\right), 7.09$ $(\mathrm{d}, 2 \mathrm{H}, J=8.4 \mathrm{~Hz}), 7.14(\mathrm{~m}, 2 \mathrm{H}), 7.27-7.37(\mathrm{~m}, 5 \mathrm{H}) .{ }^{13} \mathrm{C} \mathrm{NMR}$ $\left(\mathrm{CDCl}_{3}, 100 \mathrm{MHz}\right) \delta$, ppm: $52.1\left(\mathrm{C}^{4}\right), 120.9\left(\mathrm{q}, \mathrm{C}^{2}, J_{\mathrm{C}-\mathrm{F}}=33.6 \mathrm{~Hz}\right)$, $123.2\left(\mathrm{q}, \mathrm{C}^{1}, J_{\mathrm{C}-\mathrm{F}}=269.7 \mathrm{~Hz}\right), 127.5,128.6,129.0,129.1,130.0,133.2$, 139.7, 140.7, $141.9\left(\mathrm{q}, \mathrm{C}^{3}, J_{\mathrm{C}-\mathrm{F}}=6.3 \mathrm{~Hz}\right) .{ }^{19} \mathrm{~F} \mathrm{NMR}\left(\mathrm{CDCl}_{3}, 376\right.$ $\mathrm{MHz}) \delta$, ppm: $-63.77\left(\mathrm{dt}, \mathrm{CF}_{3}, J_{\mathrm{H}-\mathrm{F}}=6.0 \mathrm{~Hz}, J_{\mathrm{H}-\mathrm{F}}=2.0 \mathrm{~Hz}\right) . \mathrm{MS}$ (GC-MS, EI), $m / z$, (I $\left.\mathrm{I}_{\text {rel, }} \%\right): 296 / 298(25 / 9)[\mathrm{M}]^{+}, 261(100), 241$ (5), 221 (10), 183 (32), 165 (25), 149 (10), 133 (8), 115 (30), 91 (10), 51 (5). HRMS (ESI): $\mathrm{C}_{16} \mathrm{H}_{13} \mathrm{ClF}_{3}$ found $297.0656[\mathrm{M}+\mathrm{H}]^{+}$; calcd. 297.0658

(E)-1,1,1-Trifluoro-4-(4-chlorophenyl)-4-(2,4-dimethylphenyl)but-

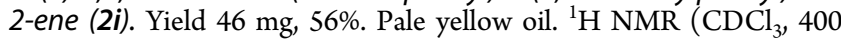
$\mathrm{MHz}) \delta$, ppm: $2.18\left(\mathrm{~s}, 3 \mathrm{H}, \mathrm{CH}_{3}\right), 2.32\left(\mathrm{~s}, 3 \mathrm{H}, \mathrm{CH}_{3}\right), 4.93-4.94(\mathrm{~m}$, $1 \mathrm{H}), 5.34\left(\mathrm{dqd}, 1 \mathrm{H}, J=15.8 \mathrm{~Hz}, J_{\mathrm{H}-\mathrm{F}}=6.3 \mathrm{~Hz}, J=1.7 \mathrm{~Hz}\right), 6.82(\mathrm{ddq}$, $\left.1 \mathrm{H}, J=15.8 \mathrm{~Hz}, J=5.9 \mathrm{~Hz}, J_{\mathrm{H}-\mathrm{F}}=2.0 \mathrm{~Hz}\right), 6.91(\mathrm{~d}, 1 \mathrm{H}, J=8.3 \mathrm{~Hz})$, $7.01(\mathrm{~m}, 2 \mathrm{H}), 7.03(\mathrm{~d}, 2 \mathrm{H}, J=8.4 \mathrm{~Hz}), 7.29(\mathrm{~d}, 2 \mathrm{H}, J=8.4 \mathrm{~Hz}) .{ }^{13} \mathrm{C}$ $\operatorname{NMR}\left(\mathrm{CDCl}_{3}, 100 \mathrm{MHz}\right) \delta$, ppm: $19.6\left(\mathrm{CH}_{3}\right), 21.1\left(\mathrm{CH}_{3}\right), 48.2\left(\mathrm{C}^{4}\right)$, $120.7\left(\mathrm{q}, \mathrm{C}^{2}, J_{\mathrm{C}-\mathrm{F}}=33.6 \mathrm{~Hz}\right), 123.3\left(\mathrm{q}, \mathrm{C}^{1}, J_{\mathrm{C}-\mathrm{F}}=269.6 \mathrm{~Hz}\right), 127.2$, $128.3,129.0,130.3,132.0,133.0,135.8,136.2,137.2,139.1,142.3(\mathrm{q}$, $\left.\mathrm{C}^{3}, J_{\mathrm{C}-\mathrm{F}}=6.3 \mathrm{~Hz}\right) .{ }^{19} \mathrm{~F} \mathrm{NMR}\left(\mathrm{CDCl}_{3}, 376 \mathrm{MHz}\right) \delta$, ppm: $-63.61(\mathrm{dt}$, $\left.\mathrm{CF}_{3}, J_{\mathrm{H}-\mathrm{F}}=6.3 \mathrm{~Hz}, J_{\mathrm{H}-\mathrm{F}}=2.2 \mathrm{~Hz}\right)$. MS (GC-MS, EI), $m / z$, ( $\left.\mathrm{I}_{\text {rel. }}, \%\right)$ : 324/326 (83/28) [M] ${ }^{+}, 309 / 311$ (100/33), 289 (52), 274 (7), 255 (8), 212 (12), 197 (19), 178 (15), 144 (16), 125 (14), 101 (16), 77 (15), 51 (5). HRMS (ESI): $\mathrm{C}_{18} \mathrm{H}_{16} \mathrm{~F}_{3} \mathrm{Cl}$ found $324.0895 \mathrm{M+}$; calcd. 324.0893.

(E)-4-(4-Chlorophenyl)-1,1,1-trifluoro-4-(3,4-dimethoxyphenyl)but-2-ene (2j). Yield $40 \mathrm{mg}, 43 \%$. Pale yellow oil. ${ }^{1} \mathrm{H} \mathrm{NMR}\left(\mathrm{CDCl}_{3}\right.$, $400 \mathrm{MHz}) \delta$, ppm: $3.82\left(\mathrm{~s}, 3 \mathrm{H}, \mathrm{CH}_{3}\right), 3.87\left(\mathrm{~s}, 3 \mathrm{H}, \mathrm{CH}_{3}\right), 4.75-4.77$ $(\mathrm{m}, 1 \mathrm{H}), 5.49\left(\mathrm{dqd}, 1 \mathrm{H}, J=15.6 \mathrm{~Hz}, J_{\mathrm{H}-\mathrm{F}}=6.0 \mathrm{~Hz}, J=1.5 \mathrm{~Hz}\right), 6.61$ $(\mathrm{d}, 1 \mathrm{H}, J=1.9 \mathrm{~Hz}), 6.67(\mathrm{dd}, 1 \mathrm{H}, J=8.2 \mathrm{~Hz}, J=1.9 \mathrm{~Hz}), 6.79(\mathrm{ddq}$, $\left.1 \mathrm{H}, J=15.6 \mathrm{~Hz}, J=6.4 \mathrm{~Hz}, J_{\mathrm{H}-\mathrm{F}}=2.0 \mathrm{~Hz}\right), 6.84(\mathrm{~d}, 1 \mathrm{H}, J=8.2 \mathrm{~Hz})$, $7.08(\mathrm{~d}, 2 \mathrm{H}, J=8.4 \mathrm{~Hz}), 7.31(\mathrm{~d}, 2 \mathrm{H}, J=8.4 \mathrm{~Hz}) .{ }^{13} \mathrm{C} \mathrm{NMR}\left(\mathrm{CDCl}_{3}\right.$, $100 \mathrm{MHz}) \delta$, ppm: $51.6\left(\mathrm{C}^{4}\right), 56.1\left(\mathrm{CH}_{3}\right), 56.1\left(\mathrm{CH}_{3}\right), 111.5,111.9$, $120.7\left(\mathrm{q}, \mathrm{C}^{2}, J_{\mathrm{C}-\mathrm{F}}=38.6 \mathrm{~Hz}\right), 120.7,123.2\left(\mathrm{q}, \mathrm{C}^{1}, J_{\mathrm{C}-\mathrm{F}}=269.6 \mathrm{~Hz}\right)$, $129.1,130.0,133.0,133.1,139.8,142.1\left(\mathrm{q}, \mathrm{C}^{3}, J_{\mathrm{C}-\mathrm{F}}=6.3 \mathrm{~Hz}\right), 148.4$, 149.4. ${ }^{19} \mathrm{~F} \mathrm{NMR}\left(\mathrm{CDCl}_{3}, 376 \mathrm{MHz}\right) \delta$, ppm: $-63.71\left(\mathrm{dt}, \mathrm{CF}_{3}, J_{\mathrm{H}-\mathrm{F}}=\right.$ $\left.6.0 \mathrm{~Hz}, J_{\mathrm{H}-\mathrm{F}}=2.0 \mathrm{~Hz}\right)$. MS (GC-MS, EI), $m / z$, $\left(\mathrm{I}_{\text {rel. }}, \%\right): 356 / 358$ $(100 / 36)[\mathrm{M}]^{+}, 341 / 343(12 / 4), 325 / 327(53 / 28), 321(10), 287$ (10), 261 (10), 213 (11), 165 (13), 138 (10), 107 (5), 89 (7), 51 (8). HRMS (ESI): $\mathrm{C}_{18} \mathrm{H}_{17} \mathrm{ClF}_{3} \mathrm{O}_{2}$ found $357.0865[\mathrm{M}+\mathrm{H}]^{+}$; calcd. 357.0869 .

(E)-1,1,1-Trifluoro-4-(3-methoxyphenyl)-4-phenylbut-2-ene (2k). Yield $34 \mathrm{mg}, 45 \%$. Pale yellow oil. ${ }^{1} \mathrm{H}$ NMR $\left(\mathrm{CDCl}_{3}, 400 \mathrm{MHz}\right) \delta$, ppm: $3.78\left(\mathrm{~s}, 1 \mathrm{H}, \mathrm{CH}_{3}\right), 4.80-4.81(\mathrm{~m}, 1 \mathrm{H}), 5.52(\mathrm{dqd}, 1 \mathrm{H}, J=15.7$ $\left.\mathrm{Hz}, J_{\mathrm{H}-\mathrm{F}}=6.3 \mathrm{~Hz}, J=1.6 \mathrm{~Hz}\right), 6.70-6.71(\mathrm{~m}, 1 \mathrm{H}), 6.76(\mathrm{~d}, 1 \mathrm{H}, J=8.6$ $\mathrm{Hz}), 6.81(\mathrm{dd}, 1 \mathrm{H}, J=8.6 \mathrm{~Hz}, J=2.3 \mathrm{~Hz}), 6.88(\mathrm{ddq}, 1 \mathrm{H}, J=15.7 \mathrm{~Hz}$, $\left.J=6.4 \mathrm{~Hz}, J_{\mathrm{H}-\mathrm{F}}=2.0 \mathrm{~Hz}\right), 7.16-7.18(\mathrm{~m}, 2 \mathrm{H}), 7.24-7.27(\mathrm{~m}, 2 \mathrm{H})$, $7.32-7.35(\mathrm{~m}, 2 \mathrm{H}) .{ }^{13} \mathrm{C} \mathrm{NMR}\left(\mathrm{CDCl}_{3}, 100 \mathrm{MHz}\right) \delta$, ppm: $52.6\left(\mathrm{C}^{4}\right)$, $55.19\left(\mathrm{CH}_{3}\right), 112.0,114.8,120.4\left(\mathrm{q}, \mathrm{C}^{2}, J_{\mathrm{C}-\mathrm{F}}=33.6 \mathrm{~Hz}\right), 120.9,123.2$ $\left(\mathrm{q}, \mathrm{C}^{1}, J_{\mathrm{C}-\mathrm{F}}=262.3 \mathrm{~Hz}\right), 127.1,128.5,128.8,129.8,140.9,142.2\left(\mathrm{q}, \mathrm{C}^{3}\right.$, $\left.J_{\mathrm{C}-\mathrm{F}}=6.4 \mathrm{~Hz}\right), 142.64,159.9 .{ }^{19} \mathrm{~F} \mathrm{NMR}\left(\mathrm{CDCl}_{3}, 376 \mathrm{MHz}\right) \delta$, ppm: $-63.70\left(\mathrm{dt}, \mathrm{CF}_{3}, J_{\mathrm{H}-\mathrm{F}}=6.3 \mathrm{~Hz}, J_{\mathrm{H}-\mathrm{F}}=2.0 \mathrm{~Hz}\right) . \mathrm{MS}(\mathrm{GC}-\mathrm{MS}, \mathrm{EI}), \mathrm{m} /$ $z,\left(\mathrm{I}_{\text {rel, }} \%\right): 292(100)[\mathrm{M}]^{+}, 277$ (9), 261 (29), 253 (5), 223 (26), 209
(19), 184 (21), 165 (40), 152 (19), 145 (21), 115 (60), 91 (24), 77 (15), 63 (15), 51 (13). HRMS (ESI): $\mathrm{C}_{17} \mathrm{H}_{16} \mathrm{~F}_{3} \mathrm{O}$ found 293.1155 [M $+\mathrm{H}]^{+}$; calcd. 293.1153 .

(E)-1,1,1-Trifluoro-4-(3-methoxyphenyl)-4-(4-methoxyphenyl)but-2-ene (2l). Yield $47 \mathrm{mg}, 56 \%$. Pale yellow oil. ${ }^{1} \mathrm{H}$ NMR $\left(\mathrm{CDCl}_{3}\right.$, $400 \mathrm{MHz}) \delta$, ppm: $3.78\left(\mathrm{~s}, 3 \mathrm{H}, \mathrm{CH}_{3}\right), 3.80\left(\mathrm{~s}, 3 \mathrm{H}, \mathrm{CH}_{3}\right), 4.75-4.77$ $(\mathrm{m}, 1 \mathrm{H}), 5.50\left(\mathrm{dqd}, 1 \mathrm{H}, J=15.6 \mathrm{~Hz}, J_{\mathrm{H}-\mathrm{F}}=6.0 \mathrm{~Hz}, J=1.5 \mathrm{~Hz}\right), 6.69$ $(\mathrm{s}, 1 \mathrm{H}), 6.75(\mathrm{~d}, 1 \mathrm{H}, J=7.9 \mathrm{~Hz}), 6.80(\mathrm{dd}, 1 \mathrm{H}, J=7.9 \mathrm{~Hz}, J=2.5 \mathrm{~Hz})$, 6.79-6.86 (m, $1 \mathrm{H}), 6.87(\mathrm{~d}, 2 \mathrm{H}, J=8.7 \mathrm{~Hz}), 7.08(\mathrm{~d}, 2 \mathrm{H}, J=8.7 \mathrm{~Hz})$, $7.25(\mathrm{t}, 1 \mathrm{H}, J=7.9 \mathrm{~Hz}) \cdot{ }^{13} \mathrm{C}$ NMR $\left(\mathrm{CDCl}_{3}, 100 \mathrm{MHz}\right) \delta$, ppm: 52.0 $\left(\mathrm{C}^{4}\right), 55.3\left(\mathrm{CH}_{3}\right), 55.4\left(\mathrm{CH}_{3}\right), 112.1,114.3,114.8,120.3\left(\mathrm{q}, \mathrm{C}^{2}, J_{\mathrm{C}-\mathrm{F}}=\right.$ $33.4 \mathrm{~Hz}), 121.0,123.3\left(\mathrm{q}, \mathrm{C}^{1}, J_{\mathrm{C}-\mathrm{F}}=269.5 \mathrm{~Hz}\right), 129.7,129.9,133.1$, $142.6\left(\mathrm{q}, \mathrm{C}^{3}, J_{\mathrm{C}-\mathrm{F}}=6.3 \mathrm{~Hz}\right), 143.1,158.8,160.0 .{ }^{19} \mathrm{~F} \mathrm{NMR}\left(\mathrm{CDCl}_{3}\right.$, $376 \mathrm{MHz}) \delta$, ppm: $-63.64\left(\mathrm{dt}, \mathrm{CF}_{3}, J_{\mathrm{H}-\mathrm{F}}=6.0 \mathrm{~Hz}, J_{\mathrm{H}-\mathrm{F}}=1.9 \mathrm{~Hz}\right) . \mathrm{MS}$ (GC-MS, EI), $m / z,\left(\mathrm{I}_{\text {rel, }} \%\right): 322(100)[\mathrm{M}]^{+}, 307$ (7), 291 (35), 279 (5), 253 (15), 239 (20), 214 (26), 195 (9), 183 (10), 165 (8), 145 (48), 141 (5), 121 (18), 103 (5), 91 (6), 77 (7), 63 (5). HRMS (ESI): $\mathrm{C}_{18} \mathrm{H}_{18} \mathrm{~F}_{3} \mathrm{O}_{2}$ found $323.1257[\mathrm{M}+\mathrm{H}]^{+}$; calcd. 323.1259.

(E)-1,1,1-Trifluoro-4-(2-methoxyphenyl)-4-(3-methoxyphenyl)but-2-ene $(2 \mathrm{~m})$. Yield $6 \mathrm{mg}, 7 \%$. Pale yellow oil. ${ }^{1} \mathrm{H}$ NMR $\left(\mathrm{CDCl}_{3}\right.$, $400 \mathrm{MHz}) \delta$, ppm: $3.77\left(\mathrm{~s}, 3 \mathrm{H}, \mathrm{CH}_{3}\right), 3.79\left(\mathrm{~s}, 3 \mathrm{H}, \mathrm{CH}_{3}\right), 5.21-5.23$ $(\mathrm{m}, 1 \mathrm{H}), 5.45$ (dqd, $\left.1 \mathrm{H}, J=15.6 \mathrm{~Hz}, J_{\mathrm{H}-\mathrm{F}}=6.4 \mathrm{~Hz}, J=0.8 \mathrm{~Hz}\right), 6.71$ $(\mathrm{s}, 1 \mathrm{H}), 6.75-6.79(\mathrm{~m}, 2 \mathrm{H}), 6.79-6.86(\mathrm{~m}, 1 \mathrm{H}), 6.89(\mathrm{~d}, 1 \mathrm{H}, J=8.1$ $\mathrm{Hz}), 6.93(\mathrm{t}, 1 \mathrm{H}, J=7.6 \mathrm{~Hz}), 7.04(\mathrm{~d}, 1 \mathrm{H}, J=7.6 \mathrm{~Hz}), 7.22(\mathrm{~d}, 1 \mathrm{H}, J=$ $8.1 \mathrm{~Hz}), 7.26(\mathrm{~m}, 1 \mathrm{H}) .{ }^{13} \mathrm{C} \mathrm{NMR}\left(\mathrm{CDCl}_{3}, 100 \mathrm{MHz}\right) \delta$, ppm: 45.7 $\left(\mathrm{C}^{4}\right), 55.3\left(\mathrm{CH}_{3}\right), 55.7\left(\mathrm{CH}_{3}\right), 111.1,111.9,115.0,120.0\left(\mathrm{q}, \mathrm{C}^{2}, J_{\mathrm{C}-\mathrm{F}}=\right.$ $33.3 \mathrm{~Hz}$ ), 120.8, 121.2, $123.5\left(\mathrm{q}, \mathrm{C}^{1}, J_{\mathrm{C}-\mathrm{F}}=269.5 \mathrm{~Hz}\right), 128.5,129.4$, $129.5,129.6,142.3\left(\mathrm{q}, \mathrm{C}^{3}, J_{\mathrm{C}-\mathrm{F}}=6.4 \mathrm{~Hz}\right), 157.0,159.8 .{ }^{19} \mathrm{~F}$ NMR $\left(\mathrm{CDCl}_{3}, 376 \mathrm{MHz}\right) \delta$, ppm: $-63.55\left(\mathrm{dt}, \mathrm{CF}_{3}, J_{\mathrm{H}-\mathrm{F}}=6.4 \mathrm{~Hz}, J_{\mathrm{H}-\mathrm{F}}=2.0\right.$ Hz). MS (GC-MS, EI), $m / z$, (I $\left.\mathrm{I}_{\text {rel, }} \%\right): 322(100)[\mathrm{M}]^{+}, 307$ (10), 291 (25), 271 (5), 251 (5), 239 (7), 225 (50), 214 (12), 197 (20), 181 (10), 165 (14), 145 (30), 131 (10), 121 (30), 107 (6), 91 (25), 77 (13), 63 (5). HRMS (ESI): $\mathrm{C}_{18} \mathrm{H}_{18} \mathrm{~F}_{3} \mathrm{O}_{2}$ found $323.1253[\mathrm{M}+\mathrm{H}]^{+}$; calcd. 323.1259.

(E)-1,1,1-Trifluoro-4-(2,5-dimethylphenyl)-4-phenylbut-2-ene (2n). Yield $40 \mathrm{mg}$, 47\%. Colorless oil. ${ }^{1} \mathrm{H} \mathrm{NMR}\left(\mathrm{CDCl}_{3}, 400 \mathrm{MHz}\right) \delta$, ppm: 2.19 (s, 3H, $\left.\mathrm{CH}_{3}\right), 2.31\left(\mathrm{~s}, 3 \mathrm{H}, \mathrm{CH}_{3}\right), 4.97-4.98(\mathrm{~m}, 1 \mathrm{H}), 5.37$ $\left(\mathrm{dqd}, 1 \mathrm{H}, J=15.6 \mathrm{~Hz}, J_{\mathrm{H}-\mathrm{F}}=6.4 \mathrm{~Hz}, J=1.2 \mathrm{~Hz}\right), 6.86(\mathrm{ddq}, 1 \mathrm{H}, J=$ $\left.15.6 \mathrm{~Hz}, J=6.0 \mathrm{~Hz}, J_{\mathrm{H}-\mathrm{F}}=2.0 \mathrm{~Hz}\right), 6.87(\mathrm{~s}, 1 \mathrm{H}), 7.01(\mathrm{~d}, 1 \mathrm{H}, J=7.8$ $\mathrm{Hz}), 7.08(\mathrm{~d}, 1 \mathrm{H}, J=7.8 \mathrm{~Hz}), 7.36(\mathrm{~d}, 2 \mathrm{H}, J=7.4 \mathrm{~Hz}), 7.24-7.34(\mathrm{~m}$, $3 \mathrm{H}) .{ }^{13} \mathrm{C}$ NMR $\left(\mathrm{CDCl}_{3}, 100 \mathrm{MHz}\right) \delta$, ppm: $19.3\left(\mathrm{CH}_{3}\right), 21.3\left(\mathrm{CH}_{3}\right)$, $49.1\left(\mathrm{C}^{4}\right), 120.5\left(\mathrm{q}, \mathrm{C}^{2}, J_{\mathrm{C}-\mathrm{F}}=33.4 \mathrm{~Hz}\right), 123.4\left(\mathrm{q}, \mathrm{C}^{1}, J_{\mathrm{C}-\mathrm{F}}=269.5 \mathrm{~Hz}\right)$, $127.1,128.0,128.8,129.0,129.1,130.9,133.3,135.9,139.1,140.1$ $142.8\left(\mathrm{q}, \mathrm{C}^{3}, J_{\mathrm{C}-\mathrm{F}}=6.3 \mathrm{~Hz}\right) .{ }^{19} \mathrm{~F}$ NMR $\left(\mathrm{CDCl}_{3}, 376 \mathrm{MHz}\right) \delta$, ppm: $-63.52\left(\mathrm{dt}, \mathrm{CF}_{3}, J_{\mathrm{H}-\mathrm{F}}=6.4 \mathrm{~Hz}, J_{\mathrm{H}-\mathrm{F}}=2.0 \mathrm{~Hz}\right)$. MS (GC-MS, EI), $m /$ $z,\left(\mathrm{I}_{\mathrm{rel},}, \%\right): 290(86)[\mathrm{M}]^{+}, 275(100), 255(6), 235(6), 212(25), 197$ (24), 193 (20), 184 (26), 178 (31), 165 (20), 143 (22), 115 (40), 103 (20), 91 (35), 77 (20), 65 (8), 51 (10). HRMS (ESI): $\mathrm{C}_{18} \mathrm{H}_{18} \mathrm{~F}_{3}$ found $291.1358[\mathrm{M}+\mathrm{H}]^{+}$; calcd. 291.1361 .

(E)-4-(4-Chlorophenyl)-1,1,1-trifluoro-4-(2,5-dimethylphenyl)but2-ene (2o). Yield $58 \mathrm{mg}$, 69\%. Pale yellow oil. ${ }^{1} \mathrm{H}$ NMR $\left(\mathrm{CDCl}_{3}, 400\right.$ $\mathrm{MHz}) \delta$, ppm: $2.17\left(\mathrm{~s}, 3 \mathrm{H}, \mathrm{CH}_{3}\right), 2.31\left(\mathrm{~s}, 3 \mathrm{H}, \mathrm{CH}_{3}\right), 4.94-4.95(\mathrm{~m}$, $1 \mathrm{H}), 5.35\left(\mathrm{dqd}, 1 \mathrm{H}, J=15.6 \mathrm{~Hz}, J_{\mathrm{H}-\mathrm{F}}=6.0 \mathrm{~Hz}, J=1.2 \mathrm{~Hz}\right), 6.79-6.85$ $(\mathrm{m}, 1 \mathrm{H}), 6.83(\mathrm{~s}, 1 \mathrm{H}), 7.01-7.09(\mathrm{~m}, 4 \mathrm{H}), 7.29(\mathrm{~d}, 2 \mathrm{H}, J=8.4 \mathrm{~Hz})$. ${ }^{13} \mathrm{C}$ NMR $\left(\mathrm{CDCl}_{3}, 100 \mathrm{MHz}\right) \delta$, ppm: $19.3\left(\mathrm{CH}_{3}\right), 21.3\left(\mathrm{CH}_{3}\right), 48.5$ $\left(\mathrm{C}^{4}\right), 120.8\left(\mathrm{q}, \mathrm{C}^{2}, J_{\mathrm{C}-\mathrm{F}}=33.6 \mathrm{~Hz}\right), 123.3\left(\mathrm{q}, \mathrm{C}^{1}, J_{\mathrm{C}-\mathrm{F}}=269.5 \mathrm{~Hz}\right)$, $128.2,128.9,129.0,130.3,131.0,133.0,133.2,136.0,138.6,139.0$, $142.3\left(\mathrm{q}, \mathrm{C}^{3}, J_{\mathrm{C}-\mathrm{F}}=6.2 \mathrm{~Hz}\right) .{ }^{19} \mathrm{~F}$ NMR $\left(\mathrm{CDCl}_{3}, 376 \mathrm{MHz}\right) \delta, \mathrm{ppm}$ : $-63.59\left(\mathrm{dt}, \mathrm{CF}_{3}, J_{\mathrm{H}-\mathrm{F}}=6.0 \mathrm{~Hz}, J_{\mathrm{H}-\mathrm{F}}=2.0 \mathrm{~Hz}\right)$. MS (GC-MS, EI), $\mathrm{m} /$ $z,\left(\mathrm{I}_{\mathrm{rel}}, \%\right): 324 / 326(87 / 31)[\mathrm{M}]^{+}, 309 / 311(100 / 33), 289(43), 274$ (10), 255 (12), 240 (7), 212 (31), 197 (25), $192(35), 178(20), 149$ (17), 143 (26), 128 (20), 115 (18), 101 (19), 77 (21), 65 (5), 51 (7). HRMS (ESI): $\mathrm{C}_{18} \mathrm{H}_{17} \mathrm{ClF}_{3}$ found $325.0965[\mathrm{M}+\mathrm{H}]^{+}$; calcd. 325.0971 .

(E)-1,1,1-Trifluoro-4-bis(4-methoxyphenyl)but-2-ene (2p). Yield $24 \mathrm{mg}, 28 \%$. Yellow oil. ${ }^{1} \mathrm{H} \mathrm{NMR}\left(\mathrm{CDCl}_{3}, 400 \mathrm{MHz}\right) \delta$, ppm: 3.82 $\left(\mathrm{s}, 6 \mathrm{H}, \mathrm{CH}_{3}\right), 4.76-4.78(\mathrm{~m}, 1 \mathrm{H}), 5.49\left(\mathrm{dqd}, 1 \mathrm{H}, J=15.6 \mathrm{~Hz}, J_{\mathrm{H}-\mathrm{F}}=\right.$ $6.4 \mathrm{~Hz}, J=1.5 \mathrm{~Hz}), 6.85\left(\mathrm{ddq}, 1 \mathrm{H}, J=15.6 \mathrm{~Hz}, J=6.8 \mathrm{~Hz}, J_{\mathrm{H}-\mathrm{F}}=2.1\right.$ $\mathrm{Hz}), 6.89(\mathrm{~d}, 4 \mathrm{H}, J=8.7 \mathrm{~Hz}), 7.09(\mathrm{~d}, 4 \mathrm{H}, J=8.7 \mathrm{~Hz}) .{ }^{13} \mathrm{C}$ NMR $\left(\mathrm{CDCl}_{3}, 100 \mathrm{MHz}\right) \delta$, ppm: $51.2\left(\mathrm{C}^{4}\right), 55.4\left(\mathrm{CH}_{3}\right), 114.2,120.0(\mathrm{q}$, $\left.\mathrm{C}^{2}, J_{\mathrm{C}-\mathrm{F}}=33.4 \mathrm{~Hz}\right), 123.4\left(\mathrm{q}, \mathrm{C}^{1}, J_{\mathrm{C}-\mathrm{F}}=269.6 \mathrm{~Hz}\right), 129.6,133.6,143.1$ 
$\left(\mathrm{q}, \mathrm{C}^{3}, J_{\mathrm{C}-\mathrm{F}}=6.2 \mathrm{~Hz}\right), 158.7 .{ }^{19} \mathrm{~F} \mathrm{NMR}\left(\mathrm{CDCl}_{3}, 376 \mathrm{MHz}\right) \delta$, ppm: $-63.57\left(\mathrm{dt}, \mathrm{CF}_{3}, J_{\mathrm{H}-\mathrm{F}}=6.4 \mathrm{~Hz}, J_{\mathrm{H}-\mathrm{F}}=2.1 \mathrm{~Hz}\right) . \mathrm{MS}(\mathrm{GC}-\mathrm{MS}, \mathrm{EI}), \mathrm{m} /$ $z$, $\left(\mathrm{I}_{\text {rel }}, \%\right): 322(100)[\mathrm{M}]^{+}, 307(5), 291(40), 253(43), 227$ (16), 214 (26), 183 (10), 165 (6), 145 (66), 121 (25), 91 (6), 77 (8), 63 (5). HRMS (ESI): $\mathrm{C}_{18} \mathrm{H}_{18} \mathrm{~F}_{3} \mathrm{O}_{2}$ found $323.1251[\mathrm{M}+\mathrm{H}]^{+}$; calcd. 323.1259 .

rel-(1S,3S)-1-(Trifluoromethyl)-4,7-dimethyl-3-phenylindane (cis3a). Yield $16 \mathrm{mg}, 19 \%$. Colorless solid, $\mathrm{mp} 101-103{ }^{\circ} \mathrm{C}(\mathrm{MeOH}) .{ }^{1} \mathrm{H}$ NMR $\left(\mathrm{CDCl}_{3}, 400 \mathrm{MHz}\right) \delta$, ppm: $2.00\left(\mathrm{~s}, 3 \mathrm{H}, \mathrm{CH}_{3}\right), 2.33-2.36(\mathrm{~m}$, $1 \mathrm{H}), 2.40\left(\mathrm{~s}, 3 \mathrm{H}, \mathrm{CH}_{3}\right), 3.00(\mathrm{dt}, 1 \mathrm{H}, J=14.4 \mathrm{~Hz}, J=10.5 \mathrm{~Hz}), 3.89$ (pseudo-quintet d, $1 \mathrm{H}, J=9.7 \mathrm{~Hz}, J_{\mathrm{H}-\mathrm{F}}=9.7 \mathrm{~Hz}, J=1.5 \mathrm{~Hz}$ ), $4.54(\mathrm{dd}$, $1 \mathrm{H}, J=10.5 \mathrm{~Hz}, J=1.5 \mathrm{~Hz}), 7.08(\mathrm{~d}, 2 \mathrm{H}, J=7.3 \mathrm{~Hz}), 7.11(\mathrm{~s}, 2 \mathrm{H})$, $7.20(\mathrm{t}, 1 \mathrm{H}, J=7.3 \mathrm{~Hz}), 7.28(\mathrm{~m}, 2 \mathrm{H}) .{ }^{13} \mathrm{C} \mathrm{NMR}\left(\mathrm{CDCl}_{3}, 100 \mathrm{MHz}\right)$ $\delta$, ppm: $19.2\left(\mathrm{CH}_{3}\right), 20.0\left(\mathrm{q}, \mathrm{CH}_{3}, J_{\mathrm{C}-\mathrm{F}}=3.6 \mathrm{~Hz}\right), 35.4\left(\mathrm{q}, \mathrm{C}^{2}, J_{\mathrm{C}-\mathrm{F}}=\right.$ $2.0 \mathrm{~Hz}), 47.9\left(\mathrm{q}, \mathrm{C}^{1}, J_{\mathrm{C}-\mathrm{F}}=28.8 \mathrm{~Hz}\right), 48.6\left(\mathrm{C}^{3}\right), 126.2,127.6\left(\mathrm{q}, \mathrm{CF}_{3}\right.$, $\left.J_{\mathrm{C}-\mathrm{F}}=280.2 \mathrm{~Hz}\right), 128.0,128.3,130.0,130.6,133.2,133.8,136.5\left(\mathrm{q}, J_{\mathrm{C}-\mathrm{F}}\right.$ $=1.8 \mathrm{~Hz}), 144.0,145.0 .{ }^{19} \mathrm{~F} \mathrm{NMR}\left(\mathrm{CDCl}_{3}, 376 \mathrm{MHz}\right) \delta$, ppm: -66.71 $\left(\mathrm{d}, \mathrm{CF}_{3}, J_{\mathrm{H}-\mathrm{F}}=9.7 \mathrm{~Hz}\right)$. MS (GC-MS, EI), $m / z$, $\left(\mathrm{I}_{\text {rel. }}, \%\right): 290(50)$ $[\mathrm{M}]^{+}, 275$ (100), $197(10), 178(5), 143(10), 115(15), 89$ (12). HRMS (ESI): $\mathrm{C}_{18} \mathrm{H}_{17} \mathrm{~F}_{3} \mathrm{Na}$ found $313.1175[\mathrm{M}+\mathrm{Na}]^{+}$; calcd. 313.1180 .

rel-(1S,3S)-3-(4-Chlorophenyl)-1-(trifluoromethyl)-4,7-dimethylindane (cis-3b). Obtained in a mixture with alkene 20 (2o:cis-3b, 1.6:1). Yield $8 \mathrm{mg}, 10 \%$. Pale yellow oil. ${ }^{1} \mathrm{H} \mathrm{NMR}\left(\mathrm{CDCl}_{3}, 400 \mathrm{MHz}\right)$ $\delta$, ppm: $1.97\left(\mathrm{~s}, 3 \mathrm{H}, \mathrm{CH}_{3}\right), 2.25-2.30(\mathrm{~m}, 1 \mathrm{H}), 2.37\left(\mathrm{~s}, 3 \mathrm{H}, \mathrm{CH}_{3}\right)$, $2.97(\mathrm{dt}, 1 \mathrm{H}, J=14.4 \mathrm{~Hz}, J=10.4 \mathrm{~Hz}$ ), 3.86 (pseudo-quintet $\mathrm{d}, 1 \mathrm{H}, J$ $\left.=9.7 \mathrm{~Hz}, J_{\mathrm{H}-\mathrm{F}}=9.7 \mathrm{~Hz}, J=1.4 \mathrm{~Hz}\right), 4.48(\mathrm{~d}, 1 \mathrm{H}, J=10.4 \mathrm{~Hz}), 6.98(\mathrm{~d}$, $2 \mathrm{H}, J=8.4 \mathrm{~Hz}), 7.09(\mathrm{~s}, 2 \mathrm{H}), 7.22(\mathrm{~d}, 2 \mathrm{H}, J=8.4 \mathrm{~Hz}) \cdot{ }^{13} \mathrm{C} \mathrm{NMR}$ $\left(\mathrm{CDCl}_{3}, 100 \mathrm{MHz}\right) \delta$, ppm: $19.2\left(\mathrm{CH}_{3}\right), 20.0\left(\mathrm{q}, \mathrm{CH}_{3}, J_{\mathrm{C}-\mathrm{F}}=3.6 \mathrm{~Hz}\right)$, $35.3\left(\mathrm{q}, \mathrm{C}^{2}, J_{\mathrm{C}-\mathrm{F}}=1.9 \mathrm{~Hz}\right), 47.8\left(\mathrm{q}, \mathrm{C}^{1}, J_{\mathrm{C}-\mathrm{F}}=28.8 \mathrm{~Hz}\right), 47.9\left(\mathrm{C}^{3}\right)$, $127.6\left(\mathrm{q}, \mathrm{CF}_{3}, J_{\mathrm{C}-\mathrm{F}}=280.3 \mathrm{~Hz}\right), 128.2,129.4,130.2,130.7,131.9$, $133.0,133.9,136.4\left(\mathrm{q}, J_{\mathrm{C}-\mathrm{F}}=2.0 \mathrm{~Hz}\right), 142.5,144.5 .{ }^{19} \mathrm{~F} \mathrm{NMR}\left(\mathrm{CDCl}_{3}\right.$, $376 \mathrm{MHz}) \delta$, ppm: $-66.78\left(\mathrm{~d}, \mathrm{CF}_{3}, J_{\mathrm{H}-\mathrm{F}}=9.7 \mathrm{~Hz}\right) . \mathrm{MS}(\mathrm{GC}-\mathrm{MS}, \mathrm{EI})$, $\mathrm{m} / z,\left(\mathrm{I}_{\text {rel, }} \%\right): 324 / 326(55 / 19)[\mathrm{M}]^{+}, 309 / 311(100 / 35), 289(30)$, 274 (5), 255 (7), 212 (10), 197 (8), 178 (5), 149 (5), 143 (10), 128 (11), 110 (6), 101 (15). HRMS (ESI): $\mathrm{C}_{18} \mathrm{H}_{17} \mathrm{ClF}_{3}$ found 325.0965 $[\mathrm{M}+\mathrm{H}]^{+}$; calcd. 325.0971 .

rel-(1S,3S)-1-(Trifluoromethyl)-4,7-dimethyl-3-(3-methylphenyl)indane (cis-3c). Yield $24 \mathrm{mg}, 28 \%$. Colorless oil. ${ }^{1} \mathrm{H} \mathrm{NMR}\left(\mathrm{CDCl}_{3}\right.$, $400 \mathrm{MHz}) \delta$, ppm: $1.98\left(\mathrm{~s}, 3 \mathrm{H}, \mathrm{CH}_{3}\right), 2.29-2.33(\mathrm{~m}, 1 \mathrm{H}), 2.30(\mathrm{~s}$, $\left.3 \mathrm{H}, \mathrm{CH}_{3}\right), 2.38\left(\mathrm{~s}, 3 \mathrm{H}, \mathrm{CH}_{3}\right), 2.97(\mathrm{dt}, 1 \mathrm{H}, J=14.4 \mathrm{~Hz}, J=10.5 \mathrm{~Hz})$, 3.86 (pseudo-quintet d, $1 \mathrm{H}, J=9.7 \mathrm{~Hz}, J_{\mathrm{H}-\mathrm{F}}=9.7 \mathrm{~Hz}, J=1.7 \mathrm{~Hz}$ ), 4.48 $(\mathrm{dd}, 1 \mathrm{H}, J=10.5 \mathrm{~Hz}, J=1.7 \mathrm{~Hz}), 6.83(\mathrm{~d}, 1 \mathrm{H}, J=7.6 \mathrm{~Hz}), 6.90(\mathrm{~s}$, $1 \mathrm{H}), 7.00(\mathrm{~d}, 1 \mathrm{H}, J=7.6 \mathrm{~Hz}), 7.09(\mathrm{~s}, 2 \mathrm{H}), 7.14(\mathrm{t}, 1 \mathrm{H}, J=7.6 \mathrm{~Hz})$. ${ }^{13} \mathrm{C}$ NMR $\left(\mathrm{CDCl}_{3}, 100 \mathrm{MHz}\right) \delta$, ppm: $19.3\left(\mathrm{CH}_{3}\right), 20.0\left(\mathrm{q}, \mathrm{CH}_{3}, J_{\mathrm{C}-\mathrm{F}}\right.$ $=3.6 \mathrm{~Hz}), 21.6\left(\mathrm{CH}_{3}\right), 35.4\left(\mathrm{q}, \mathrm{C}^{2}, J_{\mathrm{C}-\mathrm{F}}=1.9 \mathrm{~Hz}\right), 47.9\left(\mathrm{q}, \mathrm{C}^{1}, J_{\mathrm{C}-\mathrm{F}}=\right.$ $28.7 \mathrm{~Hz}), 48.6\left(\mathrm{C}^{3}\right), 125.0,126.9,127.7\left(\mathrm{q}, \mathrm{CF}_{3}, J_{\mathrm{C}-\mathrm{F}}=280.2 \mathrm{~Hz}\right)$, $128.1,128.8,129.9,130.6,133.2,133.7,136.5\left(\mathrm{q}, J_{\mathrm{C}-\mathrm{F}}=2.0 \mathrm{~Hz}\right), 137.8$, 144.0, 145.2. ${ }^{19} \mathrm{~F} \mathrm{NMR}\left(\mathrm{CDCl}_{3}, 376 \mathrm{MHz}\right) \delta$, ppm: $-66.71\left(\mathrm{~d}, \mathrm{CF}_{3}\right.$, $\left.J_{\mathrm{H}-\mathrm{F}}=9.7 \mathrm{~Hz}\right) . \mathrm{MS}(\mathrm{GC}-\mathrm{MS}, \mathrm{EI}), m / z,\left(\mathrm{I}_{\mathrm{rel}}, \%\right): 304(60)[\mathrm{M}]^{+}, 289$ (100), 212 (20), 197 (15), 143 (10), 129 (10), 110 (5), 91 (5). HRMS (ESI): $\mathrm{C}_{19} \mathrm{H}_{19} \mathrm{~F}_{3} \mathrm{Na}$ found $327.1332[\mathrm{M}+\mathrm{Na}]^{+}$; calcd. 327.1337.

rel-(1S,3S)-1-(Trifluoromethyl)-5,7-dimethyl-3-(3-methylphenyl)indane (cis-3d). Obtained in a mixture with indane trans-3d. Total yield $36 \mathrm{mg}, 42 \%$ (cis/trans ratio 1:3). Pale yellow oil. ${ }^{1} \mathrm{H}$ NMR $\left(\mathrm{CDCl}_{3}, 400 \mathrm{MHz}\right) \delta$, ppm: $2.23-2.26(\mathrm{~m}, 1 \mathrm{H}), 2.29\left(\mathrm{~s}, 3 \mathrm{H}, \mathrm{CH}_{3}\right)$, $2.35\left(\mathrm{~s}, 3 \mathrm{H}, \mathrm{CH}_{3}\right), 2.38\left(\mathrm{~s}, 3 \mathrm{H}, \mathrm{CH}_{3}\right), 2.93(\mathrm{dt}, 1 \mathrm{H}, J=14.1 \mathrm{~Hz}, J=$ $10.0 \mathrm{~Hz}$ ), 3.91 (pseudo-quintet, $1 \mathrm{H}, J=9.2 \mathrm{~Hz}, J_{\mathrm{H}-\mathrm{F}}=9.2 \mathrm{~Hz}$ ), 4.36 $(\mathrm{dd}, 1 \mathrm{H}, J=10.0 \mathrm{~Hz}, J=5.2 \mathrm{~Hz}), 6.76(\mathrm{~s}, 1 \mathrm{H}), 6.95(\mathrm{~s}, 1 \mathrm{H}), 6.97-$ $7.01(\mathrm{~m}, 2 \mathrm{H}), 7.06(\mathrm{~d}, 1 \mathrm{H}, J=8.0 \mathrm{~Hz}), 7.19-7.23(\mathrm{~m}, 1 \mathrm{H}) .{ }^{13} \mathrm{C}$ NMR $\left(\mathrm{CDCl}_{3}, 100 \mathrm{MHz}\right) \delta$, ppm: $20.4\left(\mathrm{q}, \mathrm{CH}_{3}, J_{\mathrm{C}-\mathrm{F}}=4.0 \mathrm{~Hz}\right), 21.2\left(\mathrm{CH}_{3}\right)$, $21.6\left(\mathrm{CH}_{3}\right), 35.5\left(\mathrm{q}, \mathrm{C}^{2}, J_{\mathrm{C}-\mathrm{F}}=3.4 \mathrm{~Hz}\right), 47.7\left(\mathrm{q}, \mathrm{C}^{1}, J_{\mathrm{C}-\mathrm{F}}=29.0 \mathrm{~Hz}\right)$, $49.2\left(C^{3}\right), 124.3,125.4,127.3,127.7\left(q, C_{3}, J_{C-F}=280.1 \mathrm{~Hz}\right), 128.4$, $129.1,130.6,133.1,135.8,138.1,138.9,144.9,148.0 .{ }^{19} \mathrm{~F}$ NMR $\left(\mathrm{CDCl}_{3}, 376 \mathrm{MHz}\right) \delta, \mathrm{ppm}:-66.75\left(\mathrm{~d}, \mathrm{CF}_{3}, J_{\mathrm{H}-\mathrm{F}}=9.2 \mathrm{~Hz}\right) . \mathrm{MS}$

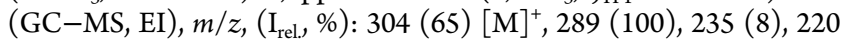
(7), 211 (7), 197 (10), 178 (5), 143 (8), 129 (10), 109 (6), 101 (5), 89 (5). HRMS (ESI): $\mathrm{C}_{19} \mathrm{H}_{19} \mathrm{~F}_{3} \mathrm{Na}$ found $327.1330[\mathrm{M}+\mathrm{Na}]^{+}$; calcd. 327.1337.
rel-(1R,3S)-1-(Trifluoromethyl)-5,7-dimethyl-3-(3-methylphenyl)indane (trans-3d). Obtained in a mixture with indane cis-3d. ${ }^{1} \mathrm{H}$ NMR $\left(\mathrm{CDCl}_{3}, 400 \mathrm{MHz}\right) \delta$, ppm: $2.23\left(\mathrm{~s}, 3 \mathrm{H}, \mathrm{CH}_{3}\right), 2.33-2.35(\mathrm{~m}, 1 \mathrm{H})$, $2.35\left(\mathrm{~s}, 3 \mathrm{H}, \mathrm{CH}_{3}\right), 2.38\left(\mathrm{~s}, 3 \mathrm{H}, \mathrm{CH}_{3}\right), 2.77(\mathrm{dd}, 1 \mathrm{H}, J=13.7 \mathrm{~Hz}, J=$ $7.2 \mathrm{~Hz}$ ), 3.91 (pseudo-quintet, $\left.1 \mathrm{H}, J=9.5 \mathrm{~Hz}, J_{\mathrm{H}-\mathrm{F}}=9.5 \mathrm{~Hz}\right), 4.52(\mathrm{dd}$, $1 \mathrm{H}, J=10.2 \mathrm{~Hz}, J=7.2 \mathrm{~Hz}), 6.56(\mathrm{~s}, 1 \mathrm{H}), 6.91(\mathrm{~s}, 1 \mathrm{H}), 6.98(\mathrm{~d}, 1 \mathrm{H}, J$ $=7.6 \mathrm{~Hz}), 7.01(\mathrm{~s}, 1 \mathrm{H}), 7.09(\mathrm{~d}, 1 \mathrm{H}, J=7.6 \mathrm{~Hz}), 7.23(\mathrm{t}, 1 \mathrm{H}, J=7.6$ $\mathrm{Hz}) .{ }^{13} \mathrm{C}$ NMR $\left(\mathrm{CDCl}_{3}, 100 \mathrm{MHz}\right) \delta$, ppm: $19.5\left(\mathrm{q}, \mathrm{CH}_{3}, J_{\mathrm{C}-\mathrm{F}}=2.7\right.$ $\mathrm{Hz}), 21.4\left(\mathrm{CH}_{3}\right), 21.6\left(\mathrm{CH}_{3}\right), 38.6\left(\mathrm{q}, \mathrm{C}^{2}, J_{\mathrm{C}-\mathrm{F}}=1.6 \mathrm{~Hz}\right), 46.8\left(\mathrm{q}, \mathrm{C}^{1}\right.$, $\left.J_{\mathrm{C}-\mathrm{F}}=27.9 \mathrm{~Hz}\right), 50.3\left(\mathrm{C}^{3}\right), 123.6,125.6,127.7,128.6\left(\mathrm{q}, \mathrm{CF}_{3}, J_{\mathrm{C}-\mathrm{F}}=\right.$ $280.9 \mathrm{~Hz}), 128.6,129.3$, 129.7, 133.1, 136.4, 138.4, 139.0, 144.4, 149.4 . ${ }^{19} \mathrm{~F} \mathrm{NMR}\left(\mathrm{CDCl}_{3}, 376 \mathrm{MHz}\right) \delta$, ppm: $-68.88\left(\mathrm{~d}, \mathrm{CF}_{3}, J_{\mathrm{H}-\mathrm{F}}=9.5 \mathrm{~Hz}\right)$. MS (GC-MS, EI), $m / z,\left(\mathrm{I}_{\text {rel, }} \%\right): 304(65)[\mathrm{M}]^{+}, 289(100), 235$ (10), 220 (5), 211 (6), 197 (10), 178 (5), 143 (10), 129 (10), 109 (6), 101 (5), 89 (5), 77 (5). HRMS (ESI): $\mathrm{C}_{19} \mathrm{H}_{19} \mathrm{~F}_{3} \mathrm{Na}$ found 327.1330 $[\mathrm{M}+\mathrm{Na}]^{+}$; calcd. 327.1337 .

rel-(1S,3S)-1-(Trifluoromethyl)-4,5,7-trimethyl-3-phenylindane (cis-3e). Yield $68 \mathrm{mg}$, 76\% (from pseudocumene), $22 \mathrm{mg}, 24 \%$ (from mesitylene). Colorless oil. ${ }^{1} \mathrm{H}$ NMR $\left(\mathrm{CDCl}_{3}, 400 \mathrm{MHz}\right) \delta$, ppm: 1.91 $\left(\mathrm{s}, 3 \mathrm{H}, \mathrm{CH}_{3}\right), 2.28\left(\mathrm{~s}, 3 \mathrm{H}, \mathrm{CH}_{3}\right), 2.32-2.36(\mathrm{~m}, 1 \mathrm{H}), 2.36(\mathrm{~s}, 3 \mathrm{H}$, $\left.\mathrm{CH}_{3}\right), 3.00(\mathrm{dt}, 1 \mathrm{H}, J=14.1 \mathrm{~Hz}, J=10.6 \mathrm{~Hz}), 3.85$ (pseudo-quintet, $\left.1 \mathrm{H}, J=9.6 \mathrm{~Hz}, J_{\mathrm{H}-\mathrm{F}}=9.6 \mathrm{~Hz}\right), 4.56(\mathrm{~d}, 1 \mathrm{H}, J=10.6 \mathrm{~Hz}), 7.02(\mathrm{~s}, 1 \mathrm{H})$, $7.07(\mathrm{~d}, 2 \mathrm{H}, J=7.4 \mathrm{~Hz}), 7.19(\mathrm{~m}, 1 \mathrm{H}), 7.27(\mathrm{~m}, 2 \mathrm{H}) .{ }^{13} \mathrm{C} \mathrm{NMR}$ $\left(\mathrm{CDCl}_{3}, 100 \mathrm{MHz}\right) \delta$, ppm: $16.2\left(\mathrm{CH}_{3}\right), 19.7\left(\mathrm{CH}_{3}\right), 19.8\left(\mathrm{q}, \mathrm{CH}_{3}\right.$, $\left.J_{\mathrm{C}-\mathrm{F}}=3.6 \mathrm{~Hz}\right), 35.7\left(\mathrm{q}, \mathrm{C}^{2}, J_{\mathrm{C}-\mathrm{F}}=1.7 \mathrm{~Hz}\right), 47.7\left(\mathrm{q}, \mathrm{C}^{1}, J_{\mathrm{C}-\mathrm{F}}=28.8 \mathrm{~Hz}\right)$, $48.8\left(\mathrm{C}^{3}\right), 126.1,127.7\left(\mathrm{q}, \mathrm{CF}_{3}, J_{\mathrm{C}-\mathrm{F}}=280.1 \mathrm{~Hz}\right), 128.0,128.2,131.6$, $131.7,133.2,134.1\left(\mathrm{q}, J_{\mathrm{C}-\mathrm{F}}=1.9 \mathrm{~Hz}\right), 137.8,144.5,145.2 .{ }^{19} \mathrm{~F}$ NMR $\left(\mathrm{CDCl}_{3}, 376 \mathrm{MHz}\right) \delta$, ppm: $-66.93\left(\mathrm{~d}, \mathrm{CF}_{3}, J_{\mathrm{H}-\mathrm{F}}=9.6 \mathrm{~Hz}\right) . \mathrm{MS}$ (GC-MS, EI), $m / z$, ( $\left.\mathrm{I}_{\text {rel. }} \%\right): 304(62)[\mathrm{M}]^{+}, 289$ (100), 235 (15), 115 (10), 91 (9). HRMS (ESI): $\mathrm{C}_{19} \mathrm{H}_{19} \mathrm{~F}_{3} \mathrm{Na}$ found $327.1330[\mathrm{M}+$ $\mathrm{Na}]^{+}$; calcd. 327.1337.

rel-(1S,3R)-1-(Trifluoromethyl)-4,5,7-trimethyl-3-phenylindane (trans-3e). Yield $30 \mathrm{mg}, 34 \%$. Colorless solid, $\mathrm{mp} 96-98^{\circ} \mathrm{C}(\mathrm{MeOH})$. ${ }^{1} \mathrm{H}$ NMR $\left(\mathrm{CDCl}_{3}, 400 \mathrm{MHz}\right) \delta$, ppm: $1.64\left(\mathrm{~s}, 3 \mathrm{H}, \mathrm{CH}_{3}\right), 2.11-2.19$ $(\mathrm{dt}, 1 \mathrm{H}, J=14.0 \mathrm{~Hz}, J=9.3 \mathrm{~Hz}), 2.19\left(\mathrm{~s}, 3 \mathrm{H}, \mathrm{CH}_{3}\right), 2.35(\mathrm{~s}, 3 \mathrm{H}$, $\mathrm{CH}_{3}$ ), 2.82 (dd, $1 \mathrm{H}, J=14.0 \mathrm{~Hz}, J=7.7 \mathrm{~Hz}$ ), 3.93 (pseudo-quintet, $1 \mathrm{H}, J=9.3 \mathrm{~Hz}, J_{\mathrm{H}-\mathrm{F}}=9.3 \mathrm{~Hz}$ ), 4.60 (pseudo-t, $1 \mathrm{H}, J=8.7 \mathrm{~Hz}$ ), $6.95(\mathrm{~s}$, $1 \mathrm{H}), 7.07(\mathrm{~d}, 2 \mathrm{H}, J=7.2 \mathrm{~Hz}), 7.21(\mathrm{t}, 1 \mathrm{H}, J=7.2 \mathrm{~Hz}), 7.28(\mathrm{t}, 2 \mathrm{H}, J=$ $7.2 \mathrm{~Hz}) \cdot{ }^{13} \mathrm{C}$ NMR $\left(\mathrm{CDCl}_{3}, 100 \mathrm{MHz}\right) \delta$, ppm: $16.2\left(\mathrm{CH}_{3}\right), 19.3(\mathrm{q}$, $\left.\mathrm{CH}_{3}, J_{\mathrm{C}-\mathrm{F}}=2.8 \mathrm{~Hz}\right), 19.9\left(\mathrm{CH}_{3}\right), 39.2\left(\mathrm{q}, \mathrm{C}^{2}, J_{\mathrm{C}-\mathrm{F}}=1.9 \mathrm{~Hz}\right), 47.0(\mathrm{q}$, $\left.\mathrm{C}^{1}, J_{\mathrm{C}-\mathrm{F}}=27.8 \mathrm{~Hz}\right), 50.6\left(\mathrm{C}^{3}\right), 126.4,127.8,128.5\left(\mathrm{q}, \mathrm{CF}_{3}, J_{\mathrm{C}-\mathrm{F}}=280.8\right.$ $\mathrm{Hz}), 131.1\left(\mathrm{C}^{6}\right), 131.2,132.4,133.9\left(\mathrm{q}, J_{\mathrm{C}-\mathrm{F}}=1.7 \mathrm{~Hz}\right), 138.2,146.2$, 146.6. ${ }^{19} \mathrm{~F}$ NMR $\left(\mathrm{CDCl}_{3}, 376 \mathrm{MHz}\right) \delta$, ppm: $-69.57\left(\mathrm{~d}, \mathrm{CF}_{3}, J_{\mathrm{H}-\mathrm{F}}=\right.$ $9.3 \mathrm{~Hz}$ ). MS (GC-MS, EI), $m / z$, ( $\left.\mathrm{I}_{\mathrm{rel}}, \%\right): 304(51)\left[\mathrm{M}^{+}, 289\right.$ (100), 235 (15), 211 (6), 183 (5), 157 (9), 115 (15), 91 (11), 77 (5). HRMS (ESI): $\mathrm{C}_{19} \mathrm{H}_{19} \mathrm{~F}_{3} \mathrm{Na}$ found $327.1330[\mathrm{M}+\mathrm{Na}]^{+}$; calcd. 327.1337.

rel-(1S,3S)-1-(Trifluoromethyl)-4,5,7-trimethyl-3-(3-methylphenyl)indane (cis-3f). Yield $52 \mathrm{mg}, 60 \%$. Pale yellow oil. ${ }^{1} \mathrm{H}$ NMR $\left(\mathrm{CDCl}_{3}, 400 \mathrm{MHz}\right) \delta$, ppm: $1.90\left(\mathrm{~s}, 3 \mathrm{H}, \mathrm{CH}_{3}\right), 2.28\left(\mathrm{~s}, 3 \mathrm{H}, \mathrm{CH}_{3}\right)$, $2.30-2.35(\mathrm{~m}, 1 \mathrm{H}), 2.31\left(\mathrm{~s}, 3 \mathrm{H}, \mathrm{CH}_{3}\right), 2.35\left(\mathrm{~s}, 3 \mathrm{H}, \mathrm{CH}_{3}\right), 2.98(\mathrm{dt}$, $1 \mathrm{H}, J=14.3 \mathrm{~Hz}, J=10.5 \mathrm{~Hz}$ ), 3.83 (pseudo-quintet, $1 \mathrm{H}, J=9.7 \mathrm{~Hz}$, $\left.J_{\mathrm{H}-\mathrm{F}}=9.7 \mathrm{~Hz}\right), 4.51(\mathrm{~d}, 1 \mathrm{H}, J=10.5 \mathrm{~Hz}), 6.82(\mathrm{~d}, 1 \mathrm{H}, J=7.6 \mathrm{~Hz}), 6.90$ $(\mathrm{s}, 1 \mathrm{H}), 7.00(\mathrm{~d}, 1 \mathrm{H}, J=7.6 \mathrm{~Hz}), 7.01(\mathrm{~s}, 1 \mathrm{H}), 7.23(\mathrm{t}, 1 \mathrm{H}, J=7.6$ $\mathrm{Hz}) .{ }^{13} \mathrm{C}$ NMR $\left(\mathrm{CDCl}_{3}, 100 \mathrm{MHz}\right) \delta$, ppm: $16.2\left(\mathrm{CH}_{3}\right), 19.7\left(\mathrm{CH}_{3}\right)$, $19.8\left(\mathrm{q}, \mathrm{CH}_{3}, J_{\mathrm{C}-\mathrm{F}}=3.5 \mathrm{~Hz}\right), 21.6\left(\mathrm{CH}_{3}\right), 35.7\left(\mathrm{q}, \mathrm{C}^{2}, J_{\mathrm{C}-\mathrm{F}}=1.9 \mathrm{~Hz}\right)$, $47.7\left(\mathrm{q}, \mathrm{C}^{1}, J_{\mathrm{C}-\mathrm{F}}=28.8 \mathrm{~Hz}\right), 48.8\left(\mathrm{C}^{3}\right), 125.1,126.8,127.7\left(\mathrm{q}, \mathrm{CF}_{3}, J_{\mathrm{C}-\mathrm{F}}\right.$ $=280.3 \mathrm{~Hz}), 128.0,128.8,131.7,131.7,133.2,134.1\left(\mathrm{q}, J_{\mathrm{C}-\mathrm{F}}=2.0 \mathrm{~Hz}\right)$, 137.7, 137.8, 144.5, 145.4. ${ }^{19} \mathrm{~F}$ NMR $\left(\mathrm{CDCl}_{3}, 376 \mathrm{MHz}\right) \delta$, ppm: $-66.94\left(\mathrm{~d}, \mathrm{CF}_{3}, J_{\mathrm{H}-\mathrm{F}}=9.7 \mathrm{~Hz}\right)$. MS (GC-MS, EI), $m / z$, ( $\left.\mathrm{I}_{\text {rel. }} \%\right): 318$ (55) $[\mathrm{M}]^{+}, 303$ (100), 249 (10), 226 (15), 211 (10), 197 (5), 157 (8), 129 (5), 105 (5). HRMS (ESI): $\mathrm{C}_{20} \mathrm{H}_{21} \mathrm{~F}_{3} \mathrm{Na}$ found $341.1489[\mathrm{M}+$ $\mathrm{Na}]^{+}$; calcd. 341.1493 .

rel-(1S,3S)-1-(Trifluoromethyl)-4,5,7-trimethyl-3-(4-methylphenyl)indane (cis-3g). Yield $66 \mathrm{mg}, 50 \%$. Colorless solid, $\mathrm{mp}$ 77-79 ${ }^{\circ} \mathrm{C}(\mathrm{MeOH}) .{ }^{1} \mathrm{H}$ NMR $\left(\mathrm{CDCl}_{3}, 400 \mathrm{MHz}\right) \delta, \mathrm{ppm}: 1.90(\mathrm{~s}, 3 \mathrm{H}$, $\left.\mathrm{CH}_{3}\right), 2.27\left(\mathrm{~s}, 3 \mathrm{H}, \mathrm{CH}_{3}\right), 2.32\left(\mathrm{~s}, 3 \mathrm{H}, \mathrm{CH}_{3}\right), 2.27-2.32(\mathrm{~m}, 1 \mathrm{H}), 2.34$ $\left(\mathrm{s}, 3 \mathrm{H}, \mathrm{CH}_{3}\right), 2.97(\mathrm{dt}, 1 \mathrm{H}, J=14.2 \mathrm{~Hz}, J=10.4 \mathrm{~Hz}$ ), 3.82 (pseudoquintet, $\left.1 \mathrm{H}, J=9.7 \mathrm{~Hz}, J_{\mathrm{H}-\mathrm{F}}=9.7 \mathrm{~Hz}\right), 4.50(\mathrm{~d}, 1 \mathrm{H}, J=10.4 \mathrm{~Hz}), 6.94$ $(\mathrm{d}, 2 \mathrm{H}, J=7.9 \mathrm{~Hz}), 7.00(\mathrm{~s}, 1 \mathrm{H}), 7.06(\mathrm{~d}, 2 \mathrm{H}, J=7.9 \mathrm{~Hz}) .{ }^{13} \mathrm{C}$ NMR $\left(\mathrm{CDCl}_{3}, 100 \mathrm{MHz}\right) \delta$, ppm: $16.2\left(\mathrm{CH}_{3}\right), 19.7\left(\mathrm{CH}_{3}\right), 19.8\left(\mathrm{q}, \mathrm{CH}_{3}\right.$, 
$\left.J_{\text {C-F }}=3.6 \mathrm{~Hz}\right), 21.2\left(\mathrm{CH}_{3}\right), 35.7\left(\mathrm{q}, \mathrm{C}^{2}, J_{\mathrm{C}-\mathrm{F}}=1.8 \mathrm{~Hz}\right), 47.7\left(\mathrm{q}, \mathrm{C}^{1}, J_{\mathrm{C}-\mathrm{F}}\right.$ $=28.8 \mathrm{~Hz}), 48.4\left(\mathrm{C}^{3}\right), 127.7\left(\mathrm{q}, \mathrm{CF}_{3}, J_{\mathrm{C}-\mathrm{F}}=280.2 \mathrm{~Hz}\right), 127.9,128.9$, $131.6,131.6,133.2,134.1\left(\mathrm{q}, J_{\mathrm{C}-\mathrm{F}}=1.9 \mathrm{~Hz}\right), 135.5,137.8,141.5,145.4$. ${ }^{19} \mathrm{~F} \mathrm{NMR}\left(\mathrm{CDCl}_{3}, 376 \mathrm{MHz}\right) \delta$, ppm: $-66.90\left(\mathrm{~d}, \mathrm{CF}_{3}, J_{\mathrm{H}-\mathrm{F}}=9.7 \mathrm{~Hz}\right)$.

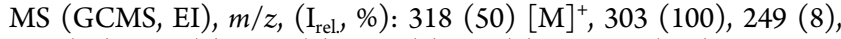
226 (25), 197 (5), 157 (8), 117 (5), 89 (5). HRMS (ESI): $\mathrm{C}_{20} \mathrm{H}_{21} \mathrm{~F}_{3}$ found $341.1490[\mathrm{M}+\mathrm{Na}]^{+}$; calcd. 341.1493 .

rel-(1S, 3R)-1-(Trifluoromethyl)-4,5,7-trimethyl-3-(4-methylphenyl)indane (trans-3g). Yield $30 \mathrm{mg}, 23 \%$. Colorless solid, $\mathrm{mp} 95-$ $97{ }^{\circ} \mathrm{C}(\mathrm{MeOH}) .{ }^{1} \mathrm{H}$ NMR $\left(\mathrm{CDCl}_{3}, 400 \mathrm{MHz}\right) \delta$, ppm: $1.66(\mathrm{~s}, 3 \mathrm{H}$, $\left.\mathrm{CH}_{3}\right), 2.13(\mathrm{dt}, 1 \mathrm{H}, J=14.0 \mathrm{~Hz}, J=9.3 \mathrm{~Hz}), 2.19\left(\mathrm{~s}, 3 \mathrm{H}, \mathrm{CH}_{3}\right), 2.33$ $\left(\mathrm{s}, 3 \mathrm{H}, \mathrm{CH}_{3}\right), 2.35\left(\mathrm{~s}, 3 \mathrm{H}, \mathrm{CH}_{3}\right), 2.79(\mathrm{dd}, 1 \mathrm{H}, J=14.0 \mathrm{~Hz}, J=7.7$ $\mathrm{Hz}$ ), 3.92 (pseudo-quintet, $1 \mathrm{H}, J=9.3 \mathrm{~Hz}, J_{\mathrm{H}-\mathrm{F}}=9.3 \mathrm{~Hz}$ ), 4.56 (pseudo-t, $1 \mathrm{H}, J=8.7 \mathrm{~Hz}), 6.94(\mathrm{~s}, 1 \mathrm{H}), 6.96(\mathrm{~d}, 2 \mathrm{H}, J=8.0 \mathrm{~Hz}), 7.09$ $(\mathrm{d}, 2 \mathrm{H}, J=8.0 \mathrm{~Hz}) \cdot{ }^{13} \mathrm{C}$ NMR $\left(\mathrm{CDCl}_{3}, 100 \mathrm{MHz}\right) \delta$, ppm: 16.2 $\left(\mathrm{CH}_{3}\right), 19.3\left(\mathrm{q}, \mathrm{CH}_{3}, J_{\mathrm{C}-\mathrm{F}}=2.8 \mathrm{~Hz}\right), 19.9\left(\mathrm{CH}_{3}\right), 21.2\left(\mathrm{CH}_{3}\right), 39.3(\mathrm{q}$, $\left.\mathrm{C}^{2}, J_{\mathrm{C}-\mathrm{F}}=1.7 \mathrm{~Hz}\right), 47.0\left(\mathrm{q}, \mathrm{C}^{1}, J_{\mathrm{C}-\mathrm{F}}=27.8 \mathrm{~Hz}\right), 50.2\left(\mathrm{C}^{3}\right), 127.7,128.5$ $\left(\mathrm{q}, \mathrm{CF}_{3}, J_{\mathrm{C}-\mathrm{F}}=280.7 \mathrm{~Hz}\right), 129.5,131.1,131.3,132.3,134.0\left(\mathrm{q}, J_{\mathrm{C}-\mathrm{F}}=\right.$ $1.7 \mathrm{~Hz}), 135.9,138.1,143.6,146.3 .{ }^{19} \mathrm{~F} \mathrm{NMR}\left(\mathrm{CDCl}_{3}, 376 \mathrm{MHz}\right) \delta$, ppm: $-69.54\left(\mathrm{~d}, \mathrm{CF}_{3}, J_{\mathrm{H}-\mathrm{F}}=9.3 \mathrm{~Hz}\right)$. MS (GC-MS, EI), $m / z$, ( $\mathrm{I}_{\text {rel }}$, \%): 318 (55) [M] $]^{+}, 303$ (100), 249 (10), 226 (20), 211 (9), 197 (5), 157 (10), 142 (5), 129 (7), 117 (7), 105 (5), 91 (5). HRMS (ESI): $\mathrm{C}_{20} \mathrm{H}_{21} \mathrm{~F}_{3} \mathrm{Na}$ found $341.1488[\mathrm{M}+\mathrm{Na}]^{+}$; calcd. 341.1493.

rel-(1S,3S)-1-(Trifluoromethyl)-4,5,7-trimethyl-3-(4-chlorophenyl)indane (cis-3h). Yield $62 \mathrm{mg}, 70 \%$ (from pseudocumene), 40 $\mathrm{mg}, 45 \%$ (from mesitylene). Pale yellow oil. ${ }^{1} \mathrm{H} \mathrm{NMR}\left(\mathrm{CDCl}_{3}, 400\right.$ $\mathrm{MHz}) \delta$, ppm: $1.90\left(\mathrm{~s}, 3 \mathrm{H}, \mathrm{CH}_{3}\right), 2.28\left(\mathrm{~s}, 3 \mathrm{H}, \mathrm{CH}_{3}\right), 2.28-2.31(\mathrm{~m}$, $1 \mathrm{H}), 2.34$ (s, $\left.3 \mathrm{H}, \mathrm{CH}_{3}\right), 2.98(\mathrm{dt}, 1 \mathrm{H}, J=14.3 \mathrm{~Hz}, J=10.4 \mathrm{~Hz}), 3.84$ (pseudo-quintet, $\left.1 \mathrm{H}, J=9.7 \mathrm{~Hz}, J_{\mathrm{H}-\mathrm{F}}=9.7 \mathrm{~Hz}\right), 4.52(\mathrm{~d}, 1 \mathrm{H}, J=10.4$ $\mathrm{Hz}), 6.99(\mathrm{~d}, 2 \mathrm{H}, J=8.4 \mathrm{~Hz}), 7.02(\mathrm{~s}, 1 \mathrm{H}), 7.23(\mathrm{~d}, 2 \mathrm{H}, J=8.4 \mathrm{~Hz})$. ${ }^{13} \mathrm{C}$ NMR $\left(\mathrm{CDCl}_{3}, 100 \mathrm{MHz}\right) \delta$, ppm: $16.2\left(\mathrm{CH}_{3}\right), 19.7\left(\mathrm{CH}_{3}\right), 19.8$ $\left(\mathrm{q}, \mathrm{CH}_{3}, J_{\mathrm{C}-\mathrm{F}}=3.4 \mathrm{~Hz}\right), 35.6\left(\mathrm{q}, \mathrm{C}^{2}, J_{\mathrm{C}-\mathrm{F}}=1.9 \mathrm{~Hz}\right), 47.6\left(\mathrm{q}, \mathrm{C}^{1}, J_{\mathrm{C}-\mathrm{F}}=\right.$ $28.8 \mathrm{~Hz}), 48.1\left(\mathrm{C}^{3}\right), 127.6\left(\mathrm{q}, \mathrm{CF}_{3}, J_{\mathrm{C}-\mathrm{F}}=280.2 \mathrm{~Hz}\right), 128.4,129.4$, $131.4,131.8,131.9,133.4,134.1\left(\mathrm{q}, J_{\mathrm{C}-\mathrm{F}}=1.9 \mathrm{~Hz}\right), 138.0,143.0,144.7$. ${ }^{19} \mathrm{~F} \mathrm{NMR}\left(\mathrm{CDCl}_{3}, 376 \mathrm{MHz}\right) \delta$, ppm: $-66.98\left(\mathrm{~d}, \mathrm{CF}_{3}, J_{\mathrm{H}-\mathrm{F}}=9.7 \mathrm{~Hz}\right)$. MS (GC-MS, EI), $m / z$, (I $\left.\mathrm{I}_{\text {rel. }} \%\right): 338 / 340(67 / 20)[\mathrm{M}]^{+}, 323 / 325$ (100/35), 303 (15), 269 (20), 254 (7), 226 (12), 143 (5), 101 (10). HRMS (ESI): $\mathrm{C}_{19} \mathrm{H}_{18} \mathrm{ClF}_{3} \mathrm{Na}$ found $361.0943[\mathrm{M}+\mathrm{Na}]^{+}$; calcd. 361.0947.

rel-(1S,3R)-1-(Trifluoromethyl)-4,5,7-trimethyl-3-(4-chlorophenyl)indane (trans-3h). Yield $32 \mathrm{mg}, 36 \%$. Pale yellow oil. ${ }^{1} \mathrm{H}$ $\operatorname{NMR}\left(\mathrm{CDCl}_{3}, 400 \mathrm{MHz}\right) \delta$, ppm: $1.65\left(\mathrm{~s}, 3 \mathrm{H}, \mathrm{CH}_{3}\right), 2.08(\mathrm{dt}, 1 \mathrm{H}, J=$ $14.0 \mathrm{~Hz}, J=9.2 \mathrm{~Hz}), 2.19\left(\mathrm{~s}, 3 \mathrm{H}, \mathrm{CH}_{3}\right), 2.34\left(\mathrm{~s}, 3 \mathrm{H}, \mathrm{CH}_{3}\right), 2.80(\mathrm{dd}$, $1 \mathrm{H}, J=14.0 \mathrm{~Hz}, J=7.7 \mathrm{~Hz}$ ), 3.62 (pseudo-quintet, $1 \mathrm{H}, J=9.2 \mathrm{~Hz}, J_{\mathrm{H}-\mathrm{F}}$ $=9.2 \mathrm{~Hz}), 4.58($ pseudo-t, $1 \mathrm{H}, J=8.6 \mathrm{~Hz}), 6.95(\mathrm{~s}, 1 \mathrm{H}), 7.00(\mathrm{~d}, 2 \mathrm{H}, J$ $=8.4 \mathrm{~Hz}), 7.25(\mathrm{~d}, 2 \mathrm{H}, J=8.4 \mathrm{~Hz}) \cdot{ }^{13} \mathrm{C} \mathrm{NMR}\left(\mathrm{CDCl}_{3}, 100 \mathrm{MHz}\right) \delta$, ppm: $16.3\left(\mathrm{CH}_{3}\right), 19.3\left(\mathrm{q}, \mathrm{CH}_{3}, J_{\mathrm{C}-\mathrm{F}}=2.6 \mathrm{~Hz}\right), 19.8\left(\mathrm{CH}_{3}\right), 39.1(\mathrm{q}$, $\left.\mathrm{C}^{2}, J_{\mathrm{C}-\mathrm{F}}=1.8 \mathrm{~Hz}\right), 47.0\left(\mathrm{q}, \mathrm{C}^{1}, J_{\mathrm{C}-\mathrm{F}}=28.0 \mathrm{~Hz}\right), 49.9\left(\mathrm{C}^{3}\right), 128.4(\mathrm{q}$, $\left.\mathrm{CF}_{3}, J_{\mathrm{C}-\mathrm{F}}=280.7 \mathrm{~Hz}\right), 129.0,129.1,131.1,131.3,132.1,132.5,133.9$ $\left(\mathrm{q}, J_{\mathrm{C}-\mathrm{F}}=1.7 \mathrm{~Hz}\right), 138.3,145.2,145.7 .{ }^{19} \mathrm{~F} \mathrm{NMR}\left(\mathrm{CDCl}_{3}, 376 \mathrm{MHz}\right) \delta$, ppm: $-69.65\left(\mathrm{~d}, \mathrm{CF}_{3}, J_{\mathrm{H}-\mathrm{F}}=9.2 \mathrm{~Hz}\right)$. MS (GC-MS, EI), $m / z$, $\left(\mathrm{I}_{\text {rel }}\right.$, \%): 338/340 (69/23) [M] $]^{+}, 323 / 325$ (100/35), 303 (10), 269 (22), 254 (9), 226 (15), 143 (8), 101 (10). HRMS (ESI): $\mathrm{C}_{19} \mathrm{H}_{18} \mathrm{ClF}_{3} \mathrm{Na}$ found $361.0943[\mathrm{M}+\mathrm{Na}]^{+}$; calcd. 361.0947 .

rel-(1S,3S)-1-(Trifluoromethyl)-4,5,7-trimethyl-3-(4-methoxyphenyl)indane (cis-3i). Yield $52 \mathrm{mg}, 60 \%$. Pale yellow oil. ${ }^{1} \mathrm{H}$ NMR $\left(\mathrm{CDCl}_{3}, 400 \mathrm{MHz}\right) \delta$, ppm: $1.90\left(\mathrm{~s}, 3 \mathrm{H}, \mathrm{CH}_{3}\right), 2.27$ (s, 3H, $\left.\mathrm{CH}_{3}\right)$, $2.27-2.31(\mathrm{~m}, 1 \mathrm{H}), 2.34\left(\mathrm{~s}, 3 \mathrm{H}, \mathrm{CH}_{3}\right), 2.95(\mathrm{dt}, 1 \mathrm{H}, J=14.3 \mathrm{~Hz}, J=$ $10.4 \mathrm{~Hz}$ ), $3.79\left(\mathrm{~s}, 3 \mathrm{H}, \mathrm{CH}_{3}\right), 3.83$ (pseudo-quintet, $1 \mathrm{H}, J=9.8 \mathrm{~Hz}, J_{\mathrm{H}-\mathrm{F}}$ $=9.8 \mathrm{~Hz}), 4.50(\mathrm{~d}, 1 \mathrm{H}, J=10.4 \mathrm{~Hz}), 6.80(\mathrm{~d}, 2 \mathrm{H}, J=8.6 \mathrm{~Hz}), 6.96(\mathrm{~d}$, $2 \mathrm{H}, J=8.6 \mathrm{~Hz}), 7.00(\mathrm{~s}, 1 \mathrm{H}) .{ }^{13} \mathrm{C} \mathrm{NMR}\left(\mathrm{CDCl}_{3}, 100 \mathrm{MHz}\right) \delta$, ppm: $16.1\left(\mathrm{CH}_{3}\right), 19.7\left(\mathrm{CH}_{3}\right), 19.8\left(\mathrm{q}, \mathrm{CH}_{3}, J_{\mathrm{C}-\mathrm{F}}=3.5 \mathrm{~Hz}\right), 35.8\left(\mathrm{q}, \mathrm{C}^{2}, J_{\mathrm{C}-\mathrm{F}}\right.$ $=1.7 \mathrm{~Hz}), 47.7\left(\mathrm{q}, \mathrm{C}^{1}, J_{\mathrm{C}-\mathrm{F}}=28.6 \mathrm{~Hz}\right), 48.0\left(\mathrm{C}^{3}\right), 55.3\left(\mathrm{CH}_{3}\right), 113.6$, $127.7\left(\mathrm{q}, \mathrm{CF}_{3}, J_{\mathrm{C}-\mathrm{F}}=280.2 \mathrm{~Hz}\right), 129.0,131.5,131.6,133.2,134.0(\mathrm{q}$, $\left.J_{\mathrm{C}-\mathrm{F}}=2.0 \mathrm{~Hz}\right), 136.7,137.8,145.6,157.9 .{ }^{19} \mathrm{~F} \mathrm{NMR}\left(\mathrm{CDCl}_{3}, 376\right.$ $\mathrm{MHz}) \delta$, ppm: $-66.86\left(\mathrm{~d}, \mathrm{CF}_{3}, J_{\mathrm{H}-\mathrm{F}}=9.8 \mathrm{~Hz}\right) . \mathrm{MS}(\mathrm{GC}-\mathrm{MS}, \mathrm{EI}), \mathrm{m} /$ $z,\left(\mathrm{I}_{\text {rel, }} \%\right): 334(73)[\mathrm{M}]^{+}, 319$ (73), 303 (10), 250 (8), 226 (100), 211 (11), 157 (23), 142 (9), 125 (15), 89 (6). HRMS (ESI): $\mathrm{C}_{20} \mathrm{H}_{21} \mathrm{~F}_{3} \mathrm{ONa}$ found $357.1439[\mathrm{M}+\mathrm{Na}]^{+}$; calcd. 357.1442.
(E)-1,1,1-Trifluoro-2,4,4-tris(4-methoxyphenyl)butane (4a). Yield $24 \mathrm{mg}, 21 \%$. Pale yellow oil. ${ }^{1} \mathrm{H}$ NMR $\left(\mathrm{CDCl}_{3}, 400 \mathrm{MHz}\right) \delta$, ppm: $2.43-2.50(\mathrm{~m}, 1 \mathrm{H}), 2.66-2.73(\mathrm{~m}, 1 \mathrm{H}), 3.00-3.10(\mathrm{~m}, 1 \mathrm{H}), 3.57(\mathrm{dd}$, $1 \mathrm{H}, J=11.9 \mathrm{~Hz}, J=3.9 \mathrm{~Hz}), 3.75\left(\mathrm{~s}, 3 \mathrm{H}, \mathrm{CH}_{3}\right), 3.81\left(\mathrm{~s}, 3 \mathrm{H}, \mathrm{CH}_{3}\right)$, $3.84\left(\mathrm{~s}, 3 \mathrm{H}, \mathrm{CH}_{3}\right), 6.79(\mathrm{~d}, 2 \mathrm{H}, J=8.6 \mathrm{~Hz}), 6.86(\mathrm{~d}, 2 \mathrm{H}, J=8.6 \mathrm{~Hz})$, $6.91(\mathrm{~d}, 2 \mathrm{H}, J=8.6 \mathrm{~Hz}), 7.04(\mathrm{~d}, 2 \mathrm{H}, J=8.4 \mathrm{~Hz}), 7.05(\mathrm{~d}, 2 \mathrm{H}, J=8.4$ $\mathrm{Hz}), 7.12(\mathrm{~d}, 2 \mathrm{H}, J=8.6 \mathrm{~Hz}) .{ }^{13} \mathrm{C} \mathrm{NMR}\left(\mathrm{CDCl}_{3}, 100 \mathrm{MHz}\right) \delta$, ppm: $34.9\left(\mathrm{C}^{3}\right), 45.9\left(\mathrm{C}^{4}\right), 47.3\left(\mathrm{q}, \mathrm{C}^{2}, J_{\text {C-F }}=26.7 \mathrm{~Hz}\right), 55.4\left(\mathrm{CH}_{3}\right), 114.0$, $114.3,114.3,126.2\left(\mathrm{q}, J_{\mathrm{C}-\mathrm{F}}=1.7 \mathrm{~Hz}\right), 127.3\left(\mathrm{q}, \mathrm{C}^{1}, J_{\mathrm{C}-\mathrm{F}}=279.2 \mathrm{~Hz}\right)$, $128.3,129.1,130.5,134.8,137.2,158.1,158.5,159.6 .{ }^{19} \mathrm{~F}$ NMR $\left(\mathrm{CDCl}_{3}, 376 \mathrm{MHz}\right) \delta$, ppm: $-69.93\left(\mathrm{~d}, \mathrm{CF}_{3}, J_{\mathrm{H}-\mathrm{F}}=9.5 \mathrm{~Hz}\right) . \mathrm{MS}$ (GC-MS, EI), $m / z$, ( $\left.\mathrm{I}_{\text {rel }}, \%\right): 430(20)\left[\mathrm{M}^{+}, 227\right.$ (100), 212 (6), 169 (5), 139 (3). HRMS (ESI): $\mathrm{C}_{25} \mathrm{H}_{26} \mathrm{~F}_{3} \mathrm{O}_{3}$ found $431.1829[\mathrm{M}+\mathrm{H}]^{+}$; calcd. 431.1834 .

(E)-2-(4,4,4-Trifluoro-1-phenylbut-2-en-1-yl)thiophene (5a). Yield $28 \mathrm{mg}, 35 \%$. Yellow oil. ${ }^{1} \mathrm{H}$ NMR $\left(\mathrm{CDCl}_{3}, 400 \mathrm{MHz}\right) \delta$, ppm: 5.03$5.04(\mathrm{~m}, 1 \mathrm{H}), 5.62\left(\mathrm{dqd}, 1 \mathrm{H}, J=15.6 \mathrm{~Hz}, J_{\mathrm{H}-\mathrm{F}}=6.2 \mathrm{~Hz}, J=1.5 \mathrm{~Hz}\right)$, $6.81-6.82(\mathrm{~m}, 1 \mathrm{H}), 6.84\left(\mathrm{ddq}, 1 \mathrm{H}, J=15.6 \mathrm{~Hz}, J=6.8 \mathrm{~Hz}, J_{\mathrm{H}-\mathrm{F}}=2.0\right.$ $\mathrm{Hz}), 6.98(\mathrm{dd}, 1 \mathrm{H}, J=5.1 \mathrm{~Hz}, J=3.5 \mathrm{~Hz}), 7.23-7.25(\mathrm{~m}, 3 \mathrm{H}), 7.29-$ $7.32 \mathrm{~m}(3 \mathrm{H}) .{ }^{13} \mathrm{C}$ NMR $\left(\mathrm{CDCl}_{3}, 100 \mathrm{MHz}\right) \delta$, ppm: $48.2\left(\mathrm{C}^{1 \prime}\right), 120.3$ $\left(\mathrm{q}, \mathrm{C}^{3 \prime}, J_{\mathrm{C}-\mathrm{F}}=33.7 \mathrm{~Hz}\right), 123.2\left(\mathrm{q}, \mathrm{C}^{4 \prime}, J_{\mathrm{C}-\mathrm{F}}=269.7 \mathrm{~Hz}\right), 125.2,125.9$, $127.1,127.7,128.3,129.0,140.9,141.8\left(\mathrm{q}, \mathrm{C}^{2 \prime}, J_{\mathrm{C}-\mathrm{F}}=6.3 \mathrm{~Hz}\right), 144.6$. ${ }^{19} \mathrm{~F} \mathrm{NMR}\left(\mathrm{CDCl}_{3}, 376 \mathrm{MHz}\right) \delta$, ppm: $-63.84\left(\mathrm{dt}, \mathrm{CF}_{3}, J_{\mathrm{H}-\mathrm{F}}=6.2 \mathrm{~Hz}\right.$, $\left.J_{\mathrm{H}-\mathrm{F}}=2.0 \mathrm{~Hz}\right) . \mathrm{MS}(\mathrm{GC}-\mathrm{MS}, \mathrm{EI}), \mathrm{m} / z,\left(\mathrm{I}_{\mathrm{rel}}, \%\right): 268(100)[\mathrm{M}]^{+}, 235$ (13), 199 (36), 184 (30), 171 (22), 165 (33), 127 (16), 115 (89), 97 (26), 77 (15), 63 (10), 45 (32), 39 (16). HRMS (ESI): $\mathrm{C}_{14} \mathrm{H}_{11} \mathrm{~F}_{3} \mathrm{SAg}$ found $374.9591[\mathrm{M}+\mathrm{Ag}]^{+}$; calcd. 374.9579 .

(E)-2-(4,4,4-Trifluoro-1-(3-methylphenyl)but-2-en-1-yl)thiophene (5b). Yield $18 \mathrm{mg}, 23 \%$. Yellow oil. ${ }^{1} \mathrm{H}$ NMR $\left(\mathrm{CDCl}_{3}, 400 \mathrm{MHz}\right) \delta$, ppm: 2.35 (s, 3H, $\left.\mathrm{CH}_{3}\right), 4.97-4.99(\mathrm{~m}, 1 \mathrm{H}), 5.61(\mathrm{dqd}, 1 \mathrm{H}, J=15.6$ $\left.\mathrm{Hz}, J_{\mathrm{H}-\mathrm{F}}=6.2 \mathrm{~Hz}, J=1.5 \mathrm{~Hz}\right), 6.81(\mathrm{~m}, 1 \mathrm{H}), 6.82(\mathrm{ddq}, 1 \mathrm{H}, J=15.6$ $\left.\mathrm{Hz}, J=6.9 \mathrm{~Hz}, J_{\mathrm{H}-\mathrm{F}}=2.0 \mathrm{~Hz}\right), 6.97(\mathrm{dd}, 1 \mathrm{H}, J=5.1 \mathrm{~Hz}, J=3.5 \mathrm{~Hz})$, $7.02-7.04(\mathrm{~m}, 2 \mathrm{H}), 7.11(\mathrm{~d}, 1 \mathrm{H}, J=7.5 \mathrm{~Hz}), 7.23(\mathrm{dd}, 1 \mathrm{H}, J=5.1 \mathrm{~Hz}$, $J=1.2 \mathrm{~Hz}), 7.25-7.27(\mathrm{~m}, 1 \mathrm{H}) .{ }^{13} \mathrm{C} \mathrm{NMR}\left(\mathrm{CDCl}_{3}, 100 \mathrm{MHz}\right) \delta$, ppm: $21.6\left(\mathrm{CH}_{3}\right), 48.2\left(\mathrm{C}^{1 \prime}\right), 120.1\left(\mathrm{q}, \mathrm{C}^{3 \prime}, J_{\mathrm{C}-\mathrm{F}}=33.6 \mathrm{~Hz}\right), 123.2(\mathrm{q}$, $\left.\mathrm{C}^{4 \prime}, J_{\mathrm{C}-\mathrm{F}}=269.6 \mathrm{~Hz}\right), 125.1,125.4,125.8,127.1,128.5,128.9,129.0$, $138.8,140.8,141.9\left(\mathrm{q}, \mathrm{C}^{21}, J_{\mathrm{C}-\mathrm{F}}=6.3 \mathrm{~Hz}\right), 144.8{ }^{19} \mathrm{~F}$ NMR $\left(\mathrm{CDCl}_{3}\right.$, $376 \mathrm{MHz}) \delta$, ppm: $-63.81\left(\mathrm{dt}, \mathrm{CF}_{3}, J_{\mathrm{H}-\mathrm{F}}=6.2 \mathrm{~Hz}, J_{\mathrm{H}-\mathrm{F}}=2.0 \mathrm{~Hz}\right) . \mathrm{MS}$ (GC-MS, EI), $m / z$, (I $\mathrm{I}_{\text {rel. }} \%$ ): $282(100)[\mathrm{M}]^{+}, 267$ (30), 249 (13), 213 (18), 198 (23), 183 (14), 165 (13), 129 (36), 121 (11), 97 (16), 65 (5), 45 (8). HRMS (ESI): $\mathrm{C}_{15} \mathrm{H}_{13} \mathrm{~F}_{3} \mathrm{SAg}$ found $388.9744[\mathrm{M}+$ $\mathrm{Ag}]^{+}$; calcd. 388.9741.

(E)-2-(4,4,4-Trifluoro-1-(4-methylphenyl)but-2-en-1-yl)thiophene (5c). Yield $24 \mathrm{mg}, 31 \%$. Yellow oil. ${ }^{1} \mathrm{H}$ NMR $\left(\mathrm{CDCl}_{3}, 400 \mathrm{MHz}\right) \delta$, ppm: 2.35 (s, $\left.3 \mathrm{H}, \mathrm{CH}_{3}\right), 4.98-5.00(\mathrm{~m}, 1 \mathrm{H}), 5.61(\mathrm{dqd}, 1 \mathrm{H}, J=15.6$ $\left.\mathrm{Hz}, J_{\mathrm{H}-\mathrm{F}}=6.2 \mathrm{~Hz}, J=1.5 \mathrm{~Hz}\right), 6.81(\mathrm{~d}, 1 \mathrm{H}, J=3.5 \mathrm{~Hz}), 6.82(\mathrm{ddq}, 1 \mathrm{H}$ $\left.J=15.6 \mathrm{~Hz}, J=6.8 \mathrm{~Hz}, J_{\mathrm{H}-\mathrm{F}}=2.0 \mathrm{~Hz}\right), 6.97(\mathrm{dd}, 1 \mathrm{H}, J=5.1 \mathrm{~Hz}, J=3.5$ $\mathrm{Hz}), 7.12(\mathrm{~d}, 2 \mathrm{H}, J=8.1 \mathrm{~Hz}), 7.17(\mathrm{~d}, 2 \mathrm{H}, J=8.1 \mathrm{~Hz}), 7.23(\mathrm{dd}, 1 \mathrm{H}, J$ $=5.1 \mathrm{~Hz}, J=1.1 \mathrm{~Hz}) \cdot{ }^{13} \mathrm{C} \operatorname{NMR}\left(\mathrm{CDCl}_{3}, 100 \mathrm{MHz}\right) \delta$, ppm: 21.2 $\left(\mathrm{CH}_{3}\right), 47.9\left(\mathrm{C}^{1 \prime}\right), 120.1\left(\mathrm{q}, \mathrm{C}^{3 \prime}, J_{\mathrm{C}-\mathrm{F}}=33.6 \mathrm{~Hz}\right), 123.2\left(\mathrm{q}, \mathrm{C}^{4 \prime}, J_{\mathrm{C}-\mathrm{F}}=\right.$ $269.7 \mathrm{~Hz}), 125.1,125.7,127.1,128.2,129.7,137.4,137.9,142.0$ (q, $\left.\mathrm{C}^{2 \prime}, J_{\mathrm{C}-\mathrm{F}}=6.4 \mathrm{~Hz}\right), 145.0 .{ }^{19} \mathrm{~F} \mathrm{NMR}\left(\mathrm{CDCl}_{3}, 376 \mathrm{MHz}\right) \delta$, ppm: $-63.81\left(\mathrm{dt}, \mathrm{CF}_{3}, J_{\mathrm{H}-\mathrm{F}}=6.2 \mathrm{~Hz}, J_{\mathrm{H}-\mathrm{F}}=2.0 \mathrm{~Hz}\right)$. MS (GC-MS, EI), $\mathrm{m} /$ $z,\left(\mathrm{I}_{\mathrm{rel},}, \%\right): 282(100)[\mathrm{M}]^{+}, 267$ (35), 249 (11), 213 (20), 198 (19), 183 (17), 165 (10), 129 (36), 115 (10), 97 (13), 45 (7). HRMS (ESI): $\mathrm{C}_{15} \mathrm{H}_{13} \mathrm{~F}_{3} \mathrm{SAg}$ found $388.9739[\mathrm{M}+\mathrm{Ag}]^{+}$; calcd. 388.9741 .

(E)-2-(1-(4-Chlorophenyl)-4,4,4-Trifluorobut-2-en-1-yl)thiophene (5d). Yield $32 \mathrm{mg}, 41 \%$. Yellow oil. ${ }^{1} \mathrm{H} \mathrm{NMR}\left(\mathrm{CDCl}_{3}, 400 \mathrm{MHz}\right) \delta$, ppm: $5.01-5.02(\mathrm{~m}, 1 \mathrm{H}), 5.61\left(\mathrm{dqd}, 1 \mathrm{H}, J=15.6 \mathrm{~Hz}, J_{\mathrm{H}-\mathrm{F}}=6.2 \mathrm{~Hz}, J\right.$ $=1.6 \mathrm{~Hz}), 6.79\left(\mathrm{ddq}, 1 \mathrm{H}, J=15.6 \mathrm{~Hz}, J=6.8 \mathrm{~Hz}, J_{\mathrm{H}-\mathrm{F}}=2.0 \mathrm{~Hz}\right), 6.81$ $(\mathrm{d}, 1 \mathrm{H}, J=3.5 \mathrm{~Hz}), 6.98(\mathrm{dd}, 1 \mathrm{H}, J=5.1 \mathrm{~Hz}, J=3.5 \mathrm{~Hz}), 7.17(\mathrm{~d}, 2 \mathrm{H}$, $J=8.5 \mathrm{~Hz}), 7.25(\mathrm{dd}, 1 \mathrm{H}, J=5.1 \mathrm{~Hz}, J=1.6 \mathrm{~Hz}), 7.33(\mathrm{~d}, 2 \mathrm{H}, J=8.5$ $\mathrm{Hz}) .{ }^{13} \mathrm{C}$ NMR $\left(\mathrm{CDCl}_{3}, 100 \mathrm{MHz}\right) \delta$, ppm: $47.5\left(\mathrm{C}^{1 \prime}\right), 120.7\left(\mathrm{q}, \mathrm{C}^{3 \prime}\right.$, $\left.J_{\mathrm{C}-\mathrm{F}}=33.9 \mathrm{~Hz}\right), 123.1\left(\mathrm{q}, \mathrm{C}^{4 \prime}, J_{\mathrm{C}-\mathrm{F}}=269.7 \mathrm{~Hz}\right), 125.4,126.0,127.2$, $129.2,129.7,133.7,139.3,141.3\left(\mathrm{q}, \mathrm{C}^{2 \prime}, J_{\mathrm{C}-\mathrm{F}}=6.3 \mathrm{~Hz}\right), 143.9 .{ }^{19} \mathrm{~F}$ $\operatorname{NMR}\left(\mathrm{CDCl}_{3}, 376 \mathrm{MHz}\right) \delta$, ppm: $-63.90\left(\mathrm{dt}, \mathrm{CF}_{3}, J_{\mathrm{H}-\mathrm{F}}=6.2 \mathrm{~Hz}, J_{\mathrm{H}-\mathrm{F}}\right.$ $=2.0 \mathrm{~Hz})$. MS (GC-MS, EI), $m / z,\left(\mathrm{I}_{\text {rel. }}, \%\right): 302 / 304(100 / 35)[\mathrm{M}]^{+}$, 267 (74), 247 (6), 233 (39), 218 (10), 198 (18), 183 (46), 171 (27), 165 (15), 149 (32), 121 (12), 97 (25), 63 (7), 45 (20). HRMS (ESI): $\mathrm{C}_{14} \mathrm{H}_{10} \mathrm{ClF}_{3} \mathrm{SAg}$ found $408.9200[\mathrm{M}+\mathrm{Ag}]^{+}$; calcd. 408.9189 . 
2,5-Bis((E)-4,4,4-trifluoro-1-phenylbut-2-en-1-yl)thiophene (6a). Yield $14 \mathrm{mg}, 10 \%$. Yellow oil. ${ }^{1} \mathrm{H}$ NMR $\left(\mathrm{CDCl}_{3}, 400 \mathrm{MHz}\right) \delta$, ppm: $4.92-4.93(\mathrm{~m}, 2 \mathrm{H}), 5.55-5.64(\mathrm{~m}, 2 \mathrm{H}), 6.65(\mathrm{~d}, 2 \mathrm{H}, J=1.5 \mathrm{~Hz})$, 6.73-6.81 (m, 2H), 7.20-7.22 (m, 4H), 7.29-7.37 (m, 6H). ${ }^{13} \mathrm{C}$ NMR $\left(\mathrm{CDCl}_{3}, 100 \mathrm{MHz}\right) \delta$, ppm: $48.4\left(\mathrm{C}^{1 \prime}\right), 120.3\left(\mathrm{q}, \mathrm{C}^{3 \prime}, J_{\mathrm{C}-\mathrm{F}}=\right.$ $33.7 \mathrm{~Hz}), 123.1\left(\mathrm{q}, \mathrm{C}^{4}, J_{\mathrm{C}-\mathrm{F}}=269.7 \mathrm{~Hz}\right), 125.7,125.7,127.8,128.3$, 129.1, 140.5, $141.5\left(\mathrm{q}, \mathrm{C}^{2 \prime}, J_{\mathrm{C}-\mathrm{F}} 6.3 \mathrm{~Hz}\right), 144.3 .{ }^{19} \mathrm{~F} \mathrm{NMR}\left(\mathrm{CDCl}_{3}, 376\right.$ $\mathrm{MHz}) \delta$, ppm: $-63.846\left(\mathrm{~m}, \mathrm{CF}_{3}\right),-63.850\left(\mathrm{~m}, \mathrm{CF}_{3}\right)$. MS (GC-MS, EI), $m / z$, (I $\left.\mathrm{I}_{\text {rel. }}, \%\right): 452(78)[\mathrm{M}]^{+}, 357$ (9), 267 (100), 183 (82), 165 (40), 133 (10), 115 (36), 91 (25), 77 (12), 45 (13). HRMS (ESI): $\mathrm{C}_{24} \mathrm{H}_{18} \mathrm{~F}_{6} \mathrm{SAg}$ found $559.0101[\mathrm{M}+\mathrm{Ag}]^{+}$; calcd. 559.0079.

2,5-Bis((E)-4,4,4-trifluoro-1-(3-methylphenyl)but-2-en-1-yl)thiophene $(6 \mathrm{~b})$. Yield $12 \mathrm{mg}, 18 \%$. Yellow oil. ${ }^{1} \mathrm{H}$ NMR $\left(\mathrm{CDCl}_{3}, 400\right.$ $\mathrm{MHz}) \delta$, ppm: $2.34\left(\mathrm{~s}, 6 \mathrm{H}, 2 \mathrm{CH}_{3}\right), 4.87-4.88(\mathrm{~m}, 2 \mathrm{H}), 5.55-5.64(\mathrm{~m}$, $2 \mathrm{H}), 6.64(\mathrm{~d}, 2 \mathrm{H}, J=0.9 \mathrm{~Hz}), 6.73-6.81(\mathrm{~m}, 2 \mathrm{H}), 7.00-7.04(\mathrm{~m}$, $4 \mathrm{H}), 7.11(\mathrm{~d}, 2 \mathrm{H}, J=7.3 \mathrm{~Hz}), 7.22-7.25(\mathrm{~m}, 2 \mathrm{H}) .{ }^{13} \mathrm{C} \mathrm{NMR}\left(\mathrm{CDCl}_{3}\right.$, $100 \mathrm{MHz}) \delta$, ppm: $21.6\left(\mathrm{CH}_{3}\right), 48.4\left(\mathrm{C}^{1 \prime}\right), 120.1\left(\mathrm{q}, \mathrm{C}^{3 \prime}, J_{\mathrm{C}-\mathrm{F}}=33.7\right.$ $\mathrm{Hz}), 123.2\left(\mathrm{q}, \mathrm{C}^{4 \prime}, J_{\mathrm{C}-\mathrm{F}}=269.7 \mathrm{~Hz}\right), 125.3,125.5,125.6,128.5,128.9$, 129.0, 138.8, $140.5,141.6\left(\mathrm{q}, \mathrm{C}^{2}, J_{\mathrm{C}-\mathrm{F}}=6.4 \mathrm{~Hz}\right), 144.4 .{ }^{19} \mathrm{~F}$ NMR $\left(\mathrm{CDCl}_{3}, 376 \mathrm{MHz}\right) \delta$, ppm: $-63.808\left(\mathrm{~m}, \mathrm{CF}_{3}\right),-63.813\left(\mathrm{~m}, \mathrm{CF}_{3}\right)$. MS (GC-MS, EI), $m / z$, ( $\left.\mathrm{I}_{\text {rel, }} \%\right): 408(82)[\mathrm{M}]^{+}, 465$ (10), 385 (8), 281 (100), 248 (7), 197 (48), 179 (12), 164 (11), 129 (11), 105 (9), 91 (4). HRMS (ESI): $\mathrm{C}_{26} \mathrm{H}_{22} \mathrm{~F}_{6} \mathrm{SAg}$ found $587.0417[\mathrm{M}+\mathrm{Ag}]^{+}$; calcd. 587.0397.

2,5-Bis((E)-4,4,4-trifluoro-1-(4-methylphenyl)but-2-en-1-yl)thiophene (6c). Yield $28 \mathrm{mg}, 42 \%$. Yellow oil. ${ }^{1} \mathrm{H}$ NMR $\left(\mathrm{CDCl}_{3}, 400\right.$ $\mathrm{MHz}) \delta$, ppm: $2.34\left(\mathrm{~s}, 6 \mathrm{H}, \mathrm{CH}_{3}\right), 4.87-4.88(\mathrm{~m}, 2 \mathrm{H}), 5.54-5.62(\mathrm{~m}$, $2 \mathrm{H}), 6.63(\mathrm{~d}, 2 \mathrm{H}, J=1.4 \mathrm{~Hz}), 6.72-6.79(\mathrm{~m}, 2 \mathrm{H}), 7.08-7.10(\mathrm{~m}$, $4 \mathrm{H}), 7.14-7.16(\mathrm{~m}, 4 \mathrm{H}) .{ }^{13} \mathrm{C}$ NMR $\left(\mathrm{CDCl}_{3}, 100 \mathrm{MHz}\right) \delta$, ppm: 21.2 $\left(\mathrm{CH}_{3}\right), 48.1\left(\mathrm{C}^{1 \prime}\right), 120.1\left(\mathrm{q}, \mathrm{C}^{3 \prime}, J_{\mathrm{C}-\mathrm{F}}=33.7 \mathrm{~Hz}\right), 125.5\left(\mathrm{q}, \mathrm{C}^{4 \prime}, J_{\mathrm{C}-\mathrm{F}}=\right.$ $269.7 \mathrm{~Hz}), 125.4,125.5,128.2,129.7,137.5,137.6,141.7\left(\mathrm{q}, \mathrm{C}^{21}, J_{\mathrm{C}-\mathrm{F}}\right.$ $=6.3 \mathrm{~Hz}), 144.5 .{ }^{19} \mathrm{~F}$ NMR $\left(\mathrm{CDCl}_{3}, 376 \mathrm{MHz}\right) \delta, \mathrm{ppm}:-63.80(\mathrm{~m}$, $\left.\mathrm{CF}_{3}\right),-63.82\left(\mathrm{~m}, \mathrm{CF}_{3}\right)$. MS (GC-MS, EI), $m / z$, (I $\left.\mathrm{I}_{\text {rel, }}, \%\right): 480(65)$ $[\mathrm{M}]^{+}, 465(14), 411(10), 385(8), 281(100), 248(8), 197(43), 179$ (15), 164 (12), 129 (16), 105 (11), 91 (5). HRMS (ESI): $\mathrm{C}_{26} \mathrm{H}_{22} \mathrm{~F}_{6} \mathrm{SAg}$ found $587.0408[\mathrm{M}+\mathrm{Ag}]^{+}$; calcd. 587.0397.

2,5-Bis((E)-1-(4-chlorophenyl)-4,4,4-trifluorobut-2-en-1-yl)thiophene $(6 d)$. Yield $12 \mathrm{mg}, 18 \%$. Yellow oil. ${ }^{1} \mathrm{H}$ NMR $\left(\mathrm{CDCl}_{3}, 400\right.$ $\mathrm{MHz}) \delta$, ppm: $4.90-4.92(\mathrm{~m}, 2 \mathrm{H}), 5.59\left(\mathrm{dqd}, 2 \mathrm{H}, J=15.6 \mathrm{~Hz}, J_{\mathrm{H}-\mathrm{F}}=\right.$ $6.3 \mathrm{~Hz}, J=1.1 \mathrm{~Hz}), 6.65(\mathrm{~d}, 2 \mathrm{H}, J=2.1 \mathrm{~Hz}), 6.74(\mathrm{ddq}, 2 \mathrm{H}, J=15.6$ $\left.\mathrm{Hz}, J=6.9 \mathrm{~Hz}, J_{\mathrm{H}-\mathrm{F}}=2.0 \mathrm{~Hz}\right), 7.13-7.15(\mathrm{~m}, 4 \mathrm{H}), 7.31-7.34(\mathrm{~m}$, $4 \mathrm{H}) .{ }^{13} \mathrm{C}$ NMR $\left(\mathrm{CDCl}_{3}, 100 \mathrm{MHz}\right) \delta$, ppm: $47.7\left(\mathrm{C}^{1 \prime}\right), 120.8\left(\mathrm{q}, \mathrm{C}^{3 \prime}\right.$, $\left.J_{\mathrm{C}-\mathrm{F}}=33.9 \mathrm{~Hz}\right), 123.0\left(\mathrm{q}, \mathrm{C}^{4 \prime}, J_{\mathrm{C}-\mathrm{F}}=269.8 \mathrm{~Hz}\right), 125.8,125.9,129.3$, 129.6, 133.8, 138.9, $140.8\left(\mathrm{q}, \mathrm{C}^{2 \prime}, J_{\mathrm{C}-\mathrm{F}}=6.3 \mathrm{~Hz}\right), 144.0 .{ }^{19} \mathrm{~F}$ NMR $\left(\mathrm{CDCl}_{3}, 376 \mathrm{MHz}\right) \delta$, ppm: $-63.926\left(\mathrm{~m}, \mathrm{CF}_{3}\right),-63.928\left(\mathrm{~m}, \mathrm{CF}_{3}\right)$. MS (GC -MS, EI), $m / z$, ( $\left.\mathrm{I}_{\text {rel, }} \%\right): 520 / 522 / 524(44 / 31 / 7)[\mathrm{M}]^{+}, 485 /$ 487 (12/4), 301/303 (100/38), 266 (8), 217 (31), 183 (9), 164 (22), 149 (9), 115 (10), 45 (6). HRMS (ESI): $\mathrm{C}_{24} \mathrm{H}_{16} \mathrm{Cl}_{2} \mathrm{~F}_{6} \mathrm{SAg}$ found $626.9312[\mathrm{M}+\mathrm{Ag}]^{+}$; calcd. 626.9299 .

\section{ASSOCIATED CONTENT}

\section{S Supporting Information}

The Supporting Information is available free of charge on the ACS Publications website at DOI: 10.1021/acs.joc.5b01398.

Crystallographic data (CIF)

${ }^{1} \mathrm{H},{ }^{13} \mathrm{C}$, and ${ }^{19} \mathrm{~F}$ spectra of compounds, X-ray data, and computational details (PDF)

\section{AUTHOR INFORMATION}

\section{Corresponding Authors}

*E-mail: nen@acylium.chem.msu.ru (V.G.N.).

*E-mail: aleksvasil@mail.ru, a.vasilyev@spbu.ru (A.V.V.).

\section{Notes}

The authors declare no competing financial interest.

\section{ACKNOWLEDGMENTS}

This work was supported by Saint Petersburg State University (grant nos. 12.50.1558.2013 and 12.38.195.2014) and RSCF (grant no. 10 14-13-00083 for V.G.N.). Spectral studies were performed at the Center for Magnetic Resonance, the Center for Chemical Analysis and Materials Research, and the Research Center for X-ray Diffraction Studies of Saint Petersburg State University.

\section{REFERENCES}

(1) See books: (a) Bégué, J. P.; Bonnet-Delpon, D.; Bioorganic and Medicinal Chemistry of Fluorine; Wiley: Hoboken, 2008. (b) Tressaud, A., Haufe, G., Eds. Fluorine and Health: Molecular Imaging, Biomedical Materials and Pharmaceuticals; Elsevier: Amsterdam, 2008; pp 553778. (c) Petrov, V. A., Ed. Fluorinated Heterocyclic Compounds: Synthesis, Chemistry, and Applications; Wiley: Hoboken, 2009. (d) Nenajdenko, V. G., Ed. Fluorine in Heterocyclic Chemistry; Springer: Berlin, 2014.

(2) See reviews: (a) Wang, J.; Sánchez-Roselló, M.; Aceña, J. L.; del Poza, C.; Sorochinsky, A. E.; Fustero, S.; Soloshonok, V. A.; Liu, H. Chem. Rev. 2014, 114, 2432-2506. (b) Ojima, I. J. Org. Chem. 2013, 78, 6358-6383. (c) Berger, R.; Resnati, G.; Metrangolo, P.; Weber, E.; Hulliger, J. Chem. Soc. Rev. 2011, 40, 3496-3508. (d) Hunter, L. Beilstein J. Org. Chem. 2010, 6, 38. (e) Ananikov, V. P.; Khemchyan, L. L.; Ivanova, Yu. V.; Bukhtiyarov, V. I.; Sorokin, A. M.; Prosvirin, I. P.; Vatsadze, S. Z.; Medved'ko, A. V.; Nuriev, V. N.; Dilman, A. D.; Levin, V. V.; Koptyug, I. V.; Kovtunov, K. V.; Zhivonitko, V. V.; Likholobov, V. A.; Romanenko, A. V.; Simonov, P. A.; Nenajdenko, V. G.; Shmatova, O. I.; Muzalevskiy, V. M.; Nechaev, M. S.; Asachenko, A. F.; Morozov, O. S.; Dzhevakov, P. B.; Osipov, S. N.; Vorobyeva, D. V.; Topchiy, M. A.; Zotova, M. A.; Ponomarenko, S. A.; Borshchev, O. V.; Luponosov, Yu. N.; Rempel, A. A.; Valeeva, A. A.; Stakheev, A. Yu.; Turova, O. V.; Mashkovsky, I. S.; Sysolyatin, S. V.; Malykhin, V. V.; Bukhtiyarova, G. A.; Terent'ev, A. O.; Krylov, I. B. Russ. Chem. Rev. 2014, 83, 885.

(3) Shimizu, M.; Takeda, Y.; Higashi, M.; Hiyama, T. Angew. Chem., Int. Ed. 2009, 48, 3653-3656.

(4) Németh, G.; Kapiller-Dezsőfi, R.; Lax, G.; Simig, G. Tetrahedron 1996, 52, 12821-12830.

(5) (a) Magarian, R. A.; Overacre, L. B.; Singh, S.; Meyer, K. L. Curr. Med. Chem. 1994, 1, 61-104. (b) Jordan, V. C. J. Med. Chem. 2003 , 46, 883-908. (c) Jordan, V. C. J. Med. Chem. 2003, 46, 1081-1090.

(6) (a) Alkhafaji, H. M. H.; Ryabukhin, D. S.; Muzalevskiy, V. M.; Vasilyev, A. V.; Fukin, G. K.; Shastin, A. V.; Nenajdenko, V. G. Eur. J. Org. Chem. 2013, 2013, 1132-1143. (b) van Alem, K.; Belder, G.; Lodder, G.; Zuilhof, H. J. Org. Chem. 2005, 70, 179-190. (c) RadixLarge, S.; Kucharski, S.; Langlois, B. R. Synthesis 2004, 36, 456-465. (d) Borisov, Yu. A. J. Struct. Chem. 2002, 43, 743-749. (e) O'Connor, M. J.; Boblak, K. N.; Topinka, M. J.; Kindelin, P. J.; Briski, J. M.; Zheng, C.; Klumpp, D. A. J. Am. Chem. Soc. 2010, 132, 3266-3267.

(7) (a) Nenajdenko, V. G.; Sanin, A. V.; Balenkova, E. S. Molecules 1997, 2, 186-232. (b) Nenajdenko, V. G.; Sanin, A. V.; Balenkova, E. S. Russ. Chem. Rev. 1999, 68, 437-458. (c) Druzhinin, S. V.; Balenkova, E. S.; Nenajdenko, V. G. Tetrahedron 2007, 63, 77537808.

(8) See review and references cited therein: Vasilyev, A. V. Russ. Chem. Rev. 2013, 82, 187-204.

(9) Kazakova, A. N.; Iakovenko, R. O.; Muzalevskiy, V. M.; Boyarskaya, I. A.; Avdontceva, M. S.; Starova, G. L.; Vasilyev, A. V.; Nenajdenko, V. G. Tetrahedron Lett. 2014, 55, 6851-6855.

(10) Baeza, A.; Najera, C. Synthesis 2014, 46, 25-34.

(11) (a) Chen, K.; Chen, H. J.; Wong, J.; Yang, J.; Pullarkat, S. A. ChemCatChem 2013, 5, 3882-3888. (b) Pallikonda, G.; Chakravarty, M. Eur. J. Org. Chem. 2013, 2013, 944-951. (c) Trillo, P.; Baeza, A.; Nájera, C. Eur. J. Org. Chem. 2012, 2012, 2929-2934. (d) Trillo, P.; Baeza, A.; Nájera, C. J. Org. Chem. 2012, 77, 7344-7354. (e) Reddy, C. R.; Jithender, E.; Krishna, G.; Reddy, G. V.; Jagadeesh, B. Org. Biomol. Chem. 2011, 9, 3940-3947. (f) Rao, W.; Chan, P. W. H. Org. 
Biomol. Chem. 2008, 6, 2426-2433. (g) Le Bras, J.; Muzart, J. Tetrahedron 2007, 63, 7942-7948. (h) Sanz, R.; Martinez, A.; Miguel, D.; Álvarez-Gutiérrez, J. M.; Rodríguez, F. Adv. Synth. Catal. 2006, 348, 1841-1845. (i) Ishino, Y.; Mihara, M.; Hayakawa, N.; Miyata, T.; Kaneko, Y.; Miyata, T. Synth. Commun. 2001, 31, 439-448. (j) Miller, W. G.; Pittman, C. U. J. Org. Chem. 1974, 39, 1955-1956.

(12) (a) Fan, G.-P.; Liu, Z.; Wang, G.-W. Green Chem. 2013, 15, 1659-1664. (b) Jana, U.; Maiti, S.; Biswas, S. Tetrahedron Lett. 2007, 48, 7160-7163. (c) Yadav, J. S.; Reddy, S. B. V.; Aravind, S.; Kumar, G. G. K. S. N.; Reddy, A. S. Tetrahedron Lett. 2007, 48, 6117-6120. (d) Yasuda, M.; Somyo, T.; Baba, A. Angew. Chem., Int. Ed. 2006, 45, 793-796.

(13) (a) Hamilton, J. Y.; Sarlah, D.; Carreira, E. M. J. Am. Chem. Soc. 2013, 135, 994-997. (b) Meng, B.; Huang, X.; Wu, L. Adv. Synth. Catal. 2013, 355, 2637-2650. (c) Meng, B.; Ma, S. Org. Lett. 2012, 14, 2674-2677. (d) Yue, H.-L.; Wei, W.; Li, M.-M.; Ji, Y.-R.; Yang, J.-X. Adv. Synth. Catal. 2011, 353, 3139-3145. (e) Wu, L.; Shi, M. Tetrahedron 2011, 67, 5732-5739. (f) Siedlecka, R. Tetrahedron 2009, $65,2351-2355$.

(14) (a) Hamilton, J. Y.; Sarlah, D.; Carreira, E. M. Angew. Chem., Int. Ed. 2013, 52, 7532-7535. (b) Ren, K.; Li, P.; Wang, L.; Zhang, X. Tetrahedron 2011, 67, 2753-2759.

(15) (a) Krautwald, S.; Sarlah, D.; Schafroth, M. A.; Carreira, E. M. Science 2013, 340, 1065-1068. (b) Xu, L.-W.; Gao, G.; Gu, F.-L.; Sheng, H.; Li, L.; Lai, G.-Q.; Jiang, J.-X. Adv. Synth. Catal. 2010, 352, 1441-1445. (c) Rao, W.; Tay, A. H. L.; Goh, P. J.; Choy, J. M. L.; Ke, J. K.; Chan, P. W. H. Tetrahedron Lett. 2008, 49, 122-126. (d) Jana, U.; Biswas, S.; Maiti, S. Tetrahedron Lett. 2007, 48, 4065-4069. (e) Maikap, G. C.; Reddy, M. M.; Mukhopadhyay, M.; Bhatia, B.; Iqbal, J. Tetrahedron 1994, 50, 9145-9156.

(16) (a) Chattaraj, P. K.; Giri, S.; Duley, S. Chem. Rev. 2011, 111, PR43-PR75. (b) Nenajdenko, V. G.; Moiseev, A. M.; Balenkova, E. S. Russ. Chem. Bull. 2004, 53, 2241-2265. (c) Nenajdenko, V. G.; Korotchenko, V. N.; Shastin, A. V.; Tyurin, D. A.; Balenkova, E. S. Russ. J. Org. Chem. 2004, 40, 1750-1756.

(17) (a) Shishido, Y.; Wakabayashi, H.; Koike, H.; Ueno, N.; Nukui, S.; Yamagishi, T.; Murata, Y.; Naganeo, F.; Mizutani, M.; Shimada, K.; Fujiwara, Y.; Sakakibara, A.; Suga, O.; Kusano, R.; Ueda, S.; Kanai, Y.; Tsuchiya, M.; Satake, K. Bioorg. Med. Chem. 2008, 16, 7193-7205. (b) Motomura, T.; Nagamori, H.; Suzawa, K.; Ito, H.; Morita, T.; Kobayashi, S.; Shinkai, H. Fluorene Compound and Pharmaceutical use Thereof. U.S. Patent 240,634, Sept. 23, 2010. (c) Ishikawa, T.; Banno, H.; Kawakita, Y.; Ohashi, T.; Kurasawa, O. Fused Heterocyclic Compound. Eur. Pat. Appl. 2,103,620, Sep. 23, 2009. (d) Iakovenko, R. O.; Kazakova, A. N.; Muzalevskiy, V. M.; Ivanov, A. Yu.; Boyarskaya, I. A.; Chicca, A.; Petrucci, V.; Gertsch, J.; Krasavin, M.; Starova, G. L.; Zolotarev, A. A.; Avdontceva, M. S.; Nenajdenko, V. G.; Vasilyev, A. V. Org. Biomol. Chem. 2015, 13, 8827-8842.

(18) (a) Aubert, C.; Bégué, J.-P.; Bonnet-Delpon, D.; Mesureur, D. J. Chem. Soc., Perkin Trans. 1 1989, 395-399. (b) Prakash, G. K. S.; Paknia, F.; Narayanan, A.; Rasul, G.; Mathew, T.; Olah, G. A. J. Fluorine Chem. 2012, 143, 292-302. (c) Abouabdellah, A.; Aubert, C.; Bégué, J.-P.; Bonnet-Delpon, D.; Guilhem, J. J. Chem. Soc., Perkin Trans. 1 1991, 1397-1403. (d) Dong, K.; Li, Y.; Wang, Zh.; Ding, K. Angew. Chem., Int. Ed. 2013, 52, 14191-14195. (e) Lorentzen, R. J.; Brewster, J. H.; Smith, H. E. J. Am. Chem. Soc. 1992, 114, 2181-2187. (f) Kundu, K.; McCullagh, J. V.; Morehead, A. T., Jr. J. Am. Chem. Soc. 2005, 127, 16042-16043. (g) O'Connor, M. J.; Boblak, K. N.; Topinka, M. J.; Kindelin, P. J.; Briski, J. M.; Zheng, Ch.; Klumpp, D. A. J. Am. Chem. Soc. 2010, 132, 3266-3267. (h) Gassman, P. G.; Ray, J. A.; Wenthold, P. G.; Mickelson, J. W. J. J. Org. Chem. 1991, 56, 51435146. (i) Furukawa, T.; Nishimine, T.; Tokunaga, E.; Hasegawa, K.; Shiro, M.; Shibata, N. Org. Lett. 2011, 13, 3972-3975.

(19) (a) Nenajdenko, V. G.; Baraznenok, I. L.; Balenkova, E. S. J. Org. Chem. 1998, 63, 6132-6136. (b) Baraznenok, I. L.; Nenajdenko, V. G.; Balenkova, E. S. Eur. J. Org. Chem. 1999, 1999, 937-941.

(20) (a) Dolomanov, O. V.; Bourhis, L. J.; Gildea, R. J.; Howard, J. A. K.; Puschmann, H. J. Appl. Crystallogr. 2009, 42, 339-341. (b)
SHELXS: Sheldrick, G. M. Acta Crystallogr., Sect. A: Found. Crystallogr. 2008, A64, 112-122.

(21) Frisch, M. J.; Trucks, G. W.; Schlegel, H. B.; Scuseria, G. E.; Robb, M. A.; Cheeseman, J. R.; Montgomery, J. A., Jr.; Vreven, T.; Kudin, K. N.; Burant, J. C.; Millam, J. M.; Iyengar, S. S.; Tomasi, J.; Barone, V.; Mennucci, B.; Cossi, M.; Scalmani, G.; Rega, N.; Petersson, G. A.; Nakatsuji, H.; Hada, M.; Ehara, M.; Toyota, K.; Fukuda, R.; Hasegawa, J.; Ishida, M.; Nakajima, T.; Honda, Y.; Kitao, O.; Nakai, H.; Klene, M.; Li, X.; Knox, J. E.; Hratchian, H. P.; Cross, J. B.; Adamo, C.; Jaramillo, J.; Gomperts, R.; Stratmann, R. E.; Yazyev, O.; Austin, A. J.; Cammi, R.; Pomelli, C.; Ochterski, J. W.; Ayala, P. Y.; Morokuma, K.; Voth, G. A.; Salvador, P.; Dannenberg, J. J.; Zakrzewski, V. G.; Dapprich, S.; Daniels, A. D.; Strain, M. C.; Farkas, O.; Malick, D. K.; Rabuck, D.; Raghavachari, K.; Foresman, J. B.; Ortiz, J. V.; Cui, Q.; Baboul, A. G.; Clifford, S.; Cioslowski, J.; Stefanov, B. B.; Liu, G.; Liashenko, A.; Piskorz, P.; Komaromi, I.; Martin, R. L.; Fox, D. J.; Keith, T.; Al-Laham, M. A.; Peng, C. Y.; Nanayakkara, A.; Challacombe, M.; Gill, P. M. W.; Johnson, B.; Chen, W.; Wong, M. W.; Gonzalez, C.; Pople, J. A. Gaussian 03, Revision B.01; Gaussian, Inc.: Pittsburgh, PA, 2003.

(22) Okano, T.; Matsubara, H.; Kusukawa, T.; Fujuta, M. J. Organomet. Chem. 2003, 676, 43-48.

(23) Shen, Ya.; Zhang, Yu.; Zhou, Yu. J. Chem. Soc., Perkin Trans. 1 1999, 1759-1763.

(24) Jiang, X.-Y.; Chu, L.; Wang, R.-W.; Qing, F.-L. Tetrahedron Lett. 2012, 53 (50), 6853-6857. 